\title{
Prevalence of Apolipoprotein E4 Genotype and Homozygotes (APOE e4/4) among Patients Diagnosed with Alzheimer's Disease: A Systematic Review and Meta-Analysis
}

\author{
Alex Ward ${ }^{a}$ Sheila Crean ${ }^{a}$ Catherine J. Mercaldi ${ }^{b}$ Jenna M. Collins ${ }^{a}$ \\ Dylan Boyd $^{\mathrm{a}}$ Michael N. Cook $^{\mathrm{c}}$ H. Michael Arrighid \\ ${ }^{a}$ Center for Epidemiology and Database Analytics, United BioSource Corporation, Lexington, Mass., ${ }^{b}$ Center for \\ Epidemiology and Database Analytics, United BioSource Corporation, Bethesda, Md., 'Pfizer Inc., Collegeville, Pa., and \\ dJANSSEN Alzheimer Immunotherapy Research and Development, LLC, South San Francisco, Calif., USA
}

\section{Key Words}

Alzheimer's disease - Apolipoprotein E - Meta-analysis •

Prevalence $\cdot$ Epidemiology

\begin{abstract}
Background: Population allele frequencies of apolipoprotein $E$ (APOE) vary by geographic region. The purpose of this study is to summarize and evaluate published estimates for the prevalence of APOE e4 carrier status among the population diagnosed with Alzheimer's disease (AD) by geographic region and country. Methods: A systematic review of English-language publications from January 1, 1985, through May 31, 2010, was conducted. Studies reporting APOE e4 status for patients diagnosed with AD were included in the analysis; trials and autopsies were excluded. APOE e4 data were pooled, and prevalence and 95\% confidence intervals (Cls) were calculated. Results: Pooled estimates for APOE e4 carrier prevalence data were derived from 142 independent samples: $48.7 \%$ (95\% Cl: 46.5-51.0), and from 73 samples for e4/4 (homozygotes): $9.6 \%$ (95\% Cl: 8.4-10.8). The highest estimates were in Northern Europe: $61.3 \%$ (95\% Cl: 55.9-66.7), e4/4 prevalence: $14.1 \%$ (95\% Cl: 12.2-16.0). The lowest estimates were in Asia and Southern Europe. Substantial hetero-
\end{abstract}

geneity of these prevalence estimates was observed. Conclusions: APOE e4 genotype prevalence varies among AD patients by region and within each country. Further exploration is warranted to better understand the substantial heterogeneity of these prevalence estimates.

Copyright $\odot 2011$ S. Karger AG, Basel

\section{Introduction}

Alzheimer's disease (AD) is the most common form of dementia, and recent prevalence estimates suggest it impacts the lives of $4.4 \%$ of the population over 65 years old [1]. Apolipoprotein E (APOE) e4 has been recognized as a genetic risk factor for developing late-onset $\mathrm{AD}$ for over two decades [2-4]. Carriers of two APOE e4 alleles (homozygotes, e4/4) have a higher risk and also earlier onset of $\mathrm{AD}$ than heterozygous carriers (e4/-) [2, 4]. However, late-onset $\mathrm{AD}$ is believed to be caused by multiple other genetic and environmental factors that are not yet completely identified, and the interactions between these are not yet well understood [5]. Some researchers have suggested contemporary environmental conditions may have led APOE e4 carriers to have an increased suscepti-

\section{KARGER}

Fax +4161306 1234 E-Mail karger@karger.ch www.karger.com

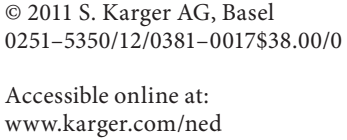

\footnotetext{
Alex Ward, PhD, MRPharmS

United BioSource Corporation

430 Bedford St., Suite 300

Lexington, MA 02420 (USA)

Tel. +1 781960 0227, E-Mail alex.ward@ unitedbiosource.com
} 
bility to developing $\mathrm{AD}$, such as high intake of carbohydrates and fat, low fiber, and reduced physical activity [6].

The APOE gene is polymorphic, with three common alleles (e2, e3, e4), and in studies of the general population, a substantial amount of variation has been consistently observed by geographic location in the six genotypes: $3 / 3,4 / 3,3 / 2,4 / 4,4 / 2$, and $2 / 2$ [7]. APOE e 3 is the most frequent [7]. APOE e4 is consistently observed to be more common in the general population residing in Northern Europe than in the Mediterranean regions of France and Italy, or in Asia [6-8]. The purpose of conducting this literature review is to summarize the published evidence on the prevalence of the APOE e4 genotype (e4/-) and homozygotes (e4/4) among patients diagnosed with AD. Specifically, published estimates of APOE e4 homozygote and heterozygote prevalence in observational studies were tabulated and reported for each country. Estimates for APOE e4 carrier prevalence were then derived for Asia, Europe (Central, North, and South/ Mediterranean), North America, and South America.

\section{Methods}

A protocol was developed and followed for each of the steps of this review, and the methods used for this review followed current practices for conducting systematic reviews and meta-analyses of the literature. The data source for this project was the literature published between January 1, 1985, and May 31, 2010. The literature search was performed using both electronic and manual components. MEDLINE (via PubMed) and EMBASE were searched to identify English-language studies published within the past 25 years. The searches were conducted using a combination of search terms and key words for apolipoproteins, APOE4, and AD and terms related to the study design, such as observational, community-based, population, cross-section, epidemiological, longitudinal, prospective, cohort studies, and cross-sectional studies. Case reports, letters, commentaries, editorials, reviews, clinical trials, meta-analyses, practice guidelines, and in vitro studies were excluded. The search terms and strategies were developed in consultation with a medical librarian, and each search strategy was adapted to the idiosyncrasies of each of these databases by using the appropriate index structures (e.g. Medical Subject Headings - MeSH in MEDLINE and EMTREE in EMBASE). The searches included limits for English language and human subjects. A manual check of the bibliographies of each of the articles included in the analysis was also performed to identify additional potentially relevant material and supplement the electronic searches.

Citations and abstracts of all studies identified in the searches were downloaded and the duplicates removed; study selection was based on 2 levels of screening. Initially, the title and abstract of each citation were screened based on the prespecified inclusion and exclusion criteria in the protocol. Articles deemed potentially eligible were then retrieved, and the full text was screened by 2 independent reviewers to determine whether it met the eligibility criteria. All disagreements were resolved by consensus.
The eligible articles were observational studies (clinic or community-based samples) with 30 or more adults diagnosed with $\mathrm{AD}$ reporting APOE e4 genotype data at the baseline of a cohort study, case-control study, or cross-sectional survey. Autopsy studies were excluded. Accepted articles describing the same AD population ('kin studies') were identified by reviewing the method section, geographic location, sample sizes, and author or institution names, and a single citation for each independent sample of patients diagnosed with $\mathrm{AD}$ was included in the analysis to avoid double-counting the results from the same population.

Data were extracted by one researcher and verified by another, and any differences were resolved by consensus. Information was extracted from each included study on country, geographic location, sources of cases (clinic, community, other), study design (cross-sectional, case control, or cohort), AD case definition, subclasses (early, late onset, sporadic, familial, mixed), and demographics (gender, race, age). The frequency of the APOE e4 genotype and, when reported, e4/4 status stratified by AD case definitions were extracted for each study.

\section{APOE e4 and e4/4 Prevalence}

Study characteristics and subject level data were first summarized using SAS version 9.1 (SAS Institute, Cary, N.C., USA) to calculate basic descriptive statistics organized by country and geographic region. The prevalences of ApoE e4 (\%) and e4/4 (\%) were computed using restricted-maximum likelihood varianceweighted random effects meta-analyses $[9,10]$ that were conducted on the APOE e 4 carrier and e $4 / 4$ proportions overall and by $\mathrm{AD}$ case definition. For this analysis, the term 'probable AD cases' is applied only to the samples specifically reported as meeting the National Institute of Neurological and Communicative Disorders and Stroke-Alzheimer's Disease and Related Disorders Association (NINCDS-ADRDA) criteria for 'probable' AD. AD cases were defined as possible when reported as 'possible' by the authors, identified as AD patients with no additional criteria, or reported as a mixed population of 'possible' and 'probable' cases.

Heterogeneity (between-study inconsistency) was investigated and measured using Cochran's Q statistic and the $\mathrm{I}^{2}$ statistic [11]. Cochran's Q, distributed as a $\chi^{2}$ statistic, estimates heterogeneity as the weighted sum of squared differences in effects across pooled studies. $\mathrm{I}^{2}$ estimates what percentage of interstudy variability is due to heterogeneity rather than to chance. As significant heterogeneity was present, the variation was investigated further by conducting stratified analyses and meta-regression. First, stratified analyses were performed by region and by country and $\mathrm{AD}$ case definition where there were at least 3 studies to pool. Next, age, AD case definition (probable AD vs. other), sample population source (community vs. other), and study design (case control vs. other), as predictors for regional APOE e 4 and e4/4 prevalence, were explored using random effects meta-regression [12]. For countries with adequate numbers of studies, the impact of sampling from $\mathrm{AD}$ populations residing in different areas within the country was also explored. If there were too few studies within a given region or country, meta-regression was not performed. Each variable was converted to binary categories and modeled simultaneously. The age category was defined as below or above the age in the lowest quartile of the average age of all the studies. Variables found to explain heterogeneity were also applied individually to regional and country estimates to explain variance. 
Fig. 1. Flow diagram of study selection.

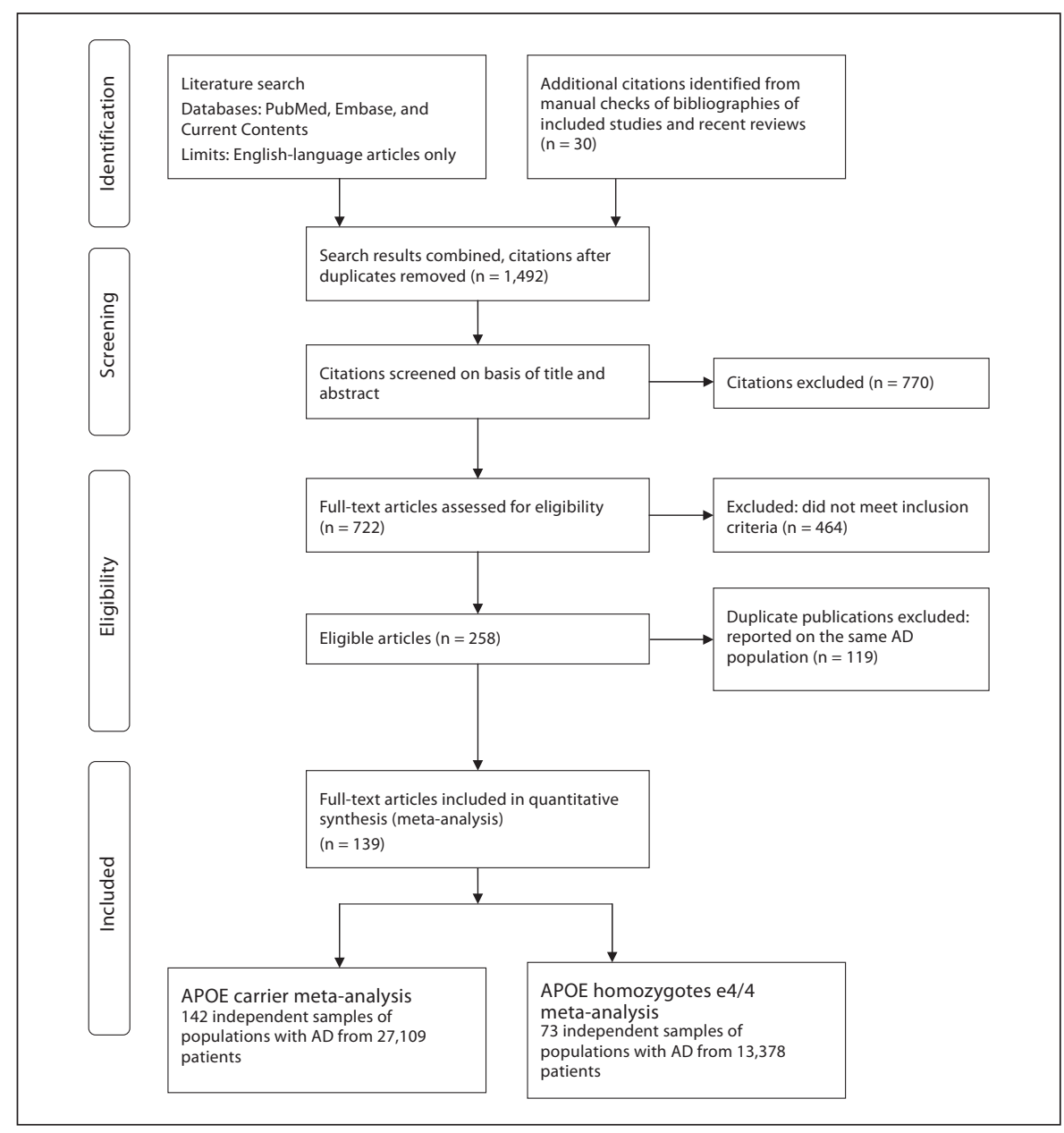

\section{Results}

\section{Study Selection}

Titles and abstracts from a total of 1,492 articles were screened, and 722 full-text articles were reviewed (see fig. 1 for additional details on each step). The reasons for exclusion were no APOE data reported or not reported separately for adult $\mathrm{AD}$ patients, study sample size fewer than 30 subjects, or autopsy study. A total of 258 articles met our eligibility criteria, of which 139 were identified as reporting APOE e4 data from 142 independent samples of patients diagnosed with AD. Four studies reported on more than 1 patient population; these groups, as well as the 'parent' studies, were considered as 2 distinct populations for the purpose of analysis. The full study listing of the 139 articles reporting the data included in the analysis is provided in the Appendix. In addition, 73 of these independent samples of patients diagnosed with $\mathrm{AD}$ also provided data on the prevalence of APOE e4/4.

\section{Study Characteristics}

The studies analyzed included a combined 27,109 individuals with a mean age of 75.2 years (range: 63.0-89.0); $64.1 \%$ were female. Fifty-eight percent $(15,773$ participants) were diagnosed as 'probable' AD cases. Thirty-one were described clearly as community-based samples (4,040 individuals), the majority were clinic- or hospitalbased samples (18,500 individuals), and the others were not possible to classify or were a combination. The analysis was conducted with separate estimation of the prevalence for 6 predefined geographic regions: Asia, Europe (Central, North, and South/Mediterranean), North America, and South America. As the region Oceania only included 1 country, Australia, this is reported with other country-specific analyses. Information from each study included in the analyses is provided for each region in tables 1-3. 
Table 1. Study characteristics and meta-analysis estimates of the prevalence of APOE e4 carriers (e4/-) and e4/4 in Asia

\begin{tabular}{|c|c|c|c|c|c|c|c|}
\hline First author, year & Country & $\begin{array}{l}\text { AD case } \\
\text { definition }\end{array}$ & $\begin{array}{l}\text { Mean } \\
\text { age } \\
\text { years }\end{array}$ & $\begin{array}{l}\mathrm{e} 4 /- \\
\mathrm{n} / \mathrm{N}\end{array}$ & $\begin{array}{l}\text { APOE e4/- } \\
\%\end{array}$ & $\begin{array}{l}\mathrm{e} 4 / 4 \\
\mathrm{n} / \mathrm{N}\end{array}$ & $\begin{array}{l}\text { APOE e } 4 / 4 \\
\%\end{array}$ \\
\hline Bi XH, 2009 & China & NR & NR & $161 / 386$ & 41.7 & NR & NR \\
\hline $\mathrm{Hu}$ J, 2009 & China & NR & 76.1 & $78 / 344$ & 22.7 & NR & NR \\
\hline Jiang H, 2005 & China & NR & 74.2 & $19 / 60$ & 31.7 & $2 / 60$ & 3.3 \\
\hline Tsai SJ, 2006 & China & NR & 74.9 & $63 / 175$ & 36.0 & NR & NR \\
\hline Wang B, 2008 & China & NR & 77.6 & $58 / 207$ & 28.0 & NR & NR \\
\hline Yang JD, 2003 & China & NR & 74.6 & $86 / 183$ & 47.0 & $23 / 183$ & 12.6 \\
\hline Zhao FG, 2005 & China & NR & 75.7 & $18 / 107$ & 16.8 & NR & NR \\
\hline Zhou S, 2010 & China & NR & 71.6 & $29 / 106$ & 27.4 & NR & NR \\
\hline Ma SL, 2005 & China & possible & 77.6 & $27 / 95$ & 28.4 & $2 / 95$ & 2.1 \\
\hline Chen L, 1999 & China & probable & NR & $73 / 196$ & 37.2 & $9 / 196$ & 4.6 \\
\hline Jiang H, 2009 & China & probable & 70.4 & $109 / 362$ & 30.1 & NR & NR \\
\hline Shi, J, 2004 & China & probable & NR & $84 / 369$ & 22.8 & NR & NR \\
\hline Tsai SJ, 2003 & China & probable & 75.8 & $85 / 234$ & 36.3 & NR & NR \\
\hline Yu JT, 2008 & China & probable & 74.2 & $49 / 109$ & 45.0 & NR & NR \\
\hline Zhang JW, 2005 & China & probable & NR & $81 / 192$ & 42.2 & NR & NR \\
\hline Chandak GR, 2002 & India & possible & NR & $17 / 49$ & 34.7 & $2 / 49$ & 4.1 \\
\hline Raygani AV, 2006 & Iran & probable & 74.2 & $34 / 94$ & 36.2 & $8 / 94$ & 8.5 \\
\hline Namekata K, 1997 & Japan & NR & NR & $127 / 294$ & 43.2 & $28 / 294$ & 9.5 \\
\hline Shibata N, 2008 & Japan & NR & 67.4 & $75 / 180$ & 41.7 & NR & NR \\
\hline Suzuki Y, 2004 & Japan & NR & 76.3 & $45 / 85$ & 52.9 & $4 / 85$ & 4.7 \\
\hline Yamagata Z, 1997 & Japan & NR & 81.6 & $79 / 163$ & 48.5 & $9 / 163$ & 5.5 \\
\hline Yoshida S, 2001 & Japan & NR & 72.6 & $17 / 32$ & 53.1 & NR & NR \\
\hline Asada T, 2000 & Japan & probable & 77.5 & $38 / 62$ & 61.3 & NR & NR \\
\hline Hu J, 1999 & Japan & probable & 73.7 & $52 / 131$ & 39.7 & $14 / 131$ & 10.7 \\
\hline Isoe K, 1996 & Japan & probable & 75.5 & $61 / 131$ & 46.6 & NR & NR \\
\hline Kawamata J, 1994 & Japan & probable & NR & $17 / 53$ & 32.1 & $4 / 53$ & 7.5 \\
\hline Kimura M, 2000 & Japan & probable & 75.1 & $121 / 216$ & 56.0 & $26 / 216$ & 12.0 \\
\hline Kimura R, 2009 & Japan & probable & 72 & $214 / 437$ & 49.0 & NR & NR \\
\hline Kuwano R, 2006 & Japan & probable & NR & $777 / 1,526$ & 50.9 & $147 / 1,526$ & 9.6 \\
\hline Matsubara-Tsutsui M, 2002 & Japan & probable & NR & $70 / 154$ & 45.5 & NR & NR \\
\hline Muramatsu T, 1996 & Japan & probable & 76 & $56 / 101$ & 55.4 & NR & NR \\
\hline Quan W, 2006 & Japan & probable & NR & $130 / 230$ & 56.5 & $32 / 230$ & 13.9 \\
\hline Tanaka N, 2010 & Japan & probable & 76.8 & $69 / 153$ & 45.1 & NR & NR \\
\hline Toji H, 1999 & Japan & probable & NR & $69 / 117$ & 59.0 & $12 / 117$ & 10.3 \\
\hline Yoshizawa T, 1997 & Japan & probable & 74.1 & $36 / 82$ & 43.9 & NR & NR \\
\hline Ponomareva NV, 2008 & Russia & probable & 65.2 & $33 / 50$ & 66.0 & $11 / 50$ & 22.0 \\
\hline Jo SA, 2006 & South Korea & probable & 73 & $157 / 316$ & 49.7 & NR & NR \\
\hline Kim HC, 2000 & South Korea & probable & 71 & $13 / 30$ & 43.3 & $1 / 30$ & 3.3 \\
\hline Kim JM, 2002 & South Korea & probable & 76.5 & $18 / 52$ & 34.6 & $1 / 52$ & 1.9 \\
\hline Kim JM, 2002 & South Korea & probable & 77.4 & $27 / 52$ & 51.9 & $1 / 52$ & 1.9 \\
\hline Huang H, 2002 & Taiwan & NR & 76.3 & $32 / 99$ & 32.3 & $6 / 99$ & 6.1 \\
\hline Liu HC, 2005 & Taiwan & $\mathrm{NR}$ & 75.5 & $83 / 232$ & 35.8 & NR & NR \\
\hline Senanarong V, 2001 & Thailand & probable & NR & $25 / 42$ & 59.5 & $5 / 42$ & 11.9 \\
\hline MA estimate (95\% CI) & & & & $43(8,288)$ & $\begin{array}{l}41.88 \\
(38.48-45.27)^{*}\end{array}$ & $21(3,817)$ & $\begin{array}{l}7.70 \\
(5.84-9.55)^{*}\end{array}$ \\
\hline \multicolumn{8}{|l|}{ Test for heterogeneity } \\
\hline $\mathrm{I}^{2}$ & & & & $89 \%$ & & $61.00 \%$ & \\
\hline Square-root Tau & & & & 10.38 & & 3.46 & \\
\hline Q p value & & & & $<0.001$ & & $<0.001$ & \\
\hline
\end{tabular}

* Significant heterogeneity at $\mathrm{p}<0.01 . \mathrm{NR}=$ Not reported. 
Table 2. Study characteristics and meta-analysis estimates of the prevalence of APOE e4 carriers (e4/-) and e4/4 in Europe

\begin{tabular}{|c|c|c|c|c|c|c|c|}
\hline First author, year & Country & $\begin{array}{l}\text { AD case } \\
\text { definition }\end{array}$ & $\begin{array}{l}\text { Mean } \\
\text { age } \\
\text { years }\end{array}$ & $\begin{array}{l}\mathrm{e} 4 /- \\
\mathrm{n} / \mathrm{N}\end{array}$ & $\begin{array}{l}\text { APOE e4/- } \\
\%\end{array}$ & $\begin{array}{l}\mathrm{e} 4 / 4 \\
\mathrm{n} / \mathrm{N}\end{array}$ & $\begin{array}{l}\mathrm{e} 4 / 4 \\
\%\end{array}$ \\
\hline \multicolumn{8}{|l|}{ Northern Europe } \\
\hline Kuusisto J, 1994 & Finland & possible & NR & $27 / 46$ & 58.7 & $6 / 46$ & 13.0 \\
\hline Tilvis RS, 1998 & Finland & possible & NR & $21 / 41$ & 51.2 & NR & NR \\
\hline Vepsalainen S, 2007 & Finland & probable & NR & $312 / 424$ & 73.6 & NR & NR \\
\hline Yasuda M, 1998 & Finland & probable & 74.5 & $116 / 178$ & 65.2 & $23 / 178$ & 12.9 \\
\hline Slooter AJC, 1998 & The Netherlands & NR & 84.1 & $43 / 129$ & 33.3 & $7 / 129$ & 5.4 \\
\hline van der Flier WM, 2008 & The Netherlands & NR & 69 & $175 / 251$ & 69.7 & $38 / 251$ & 15.1 \\
\hline van Duijn CM, 1994 & The Netherlands & NR & 63 & $92 / 175$ & 52.6 & $29 / 175$ & 16.6 \\
\hline Sando SB, 2008 & Norway & possible & 79.5 & $241 / 376$ & 64.1 & $57 / 376$ & 15.2 \\
\hline Lilius L, 1999 & Sweden & $\mathrm{NR}$ & NR & $44 / 94$ & 46.8 & NR & NR \\
\hline Prince JA, 2004 & Sweden & NR & 77.4 & $367 / 563$ & 65.2 & $70 / 563$ & 12.4 \\
\hline Andreasen N, 1999 & Sweden & probable & 71.4 & $36 / 52$ & 69.2 & NR & NR \\
\hline Landen M, 1996 & Sweden & probable & NR & $45 / 54$ & 83.3 & $12 / 54$ & 22.2 \\
\hline Lilius L, 1999 & Sweden & probable & NR & $130 / 175$ & 74.3 & NR & NR \\
\hline Zetterberg M, 2008 & Sweden & probable & 75.8 & $548 / 800$ & 68.5 & $135 / 800$ & 16.9 \\
\hline Davidson Y, 2007 & UK & NR & 63.1 & $291 / 462$ & 63.0 & $73 / 462$ & 15.8 \\
\hline Holmes C, 1996 & UK & possible & 81.9 & $92 / 164$ & 56.1 & NR & NR \\
\hline Craig D, 2005 & UK & probable & 78 & $221 / 404$ & 54.7 & $49 / 404$ & 12.1 \\
\hline McIlroy SP, 1999 & UK & probable & 77.7 & $86 / 175$ & 49.1 & NR & NR \\
\hline MA estimate (95\% CI) & & & & $18(4,563)$ & $\begin{array}{l}61.25 \\
(55.85-66.66)^{* *}\end{array}$ & $11(3,438)$ & $\begin{array}{l}14.10 \\
(12.19-16.02)^{*}\end{array}$ \\
\hline $\begin{array}{l}\text { Test for heterogeneity } \\
\mathrm{I}^{2} \\
\text { Square-root Tau } \\
\text { Q p value }\end{array}$ & & & & $\begin{array}{l}89.00 \% \\
10.96 \\
<0.001\end{array}$ & & $\begin{array}{l}51.00 \% \\
2.31 \\
0.025\end{array}$ & \\
\hline \multicolumn{8}{|l|}{ Central Europe } \\
\hline Grunblatt E, 2008 & Austria & possible & NR & $33 / 127$ & 26.0 & NR & NR \\
\hline Bettens K, 2009 & Belgium & probable & NR & $286 / 555$ & 51.5 & NR & NR \\
\hline Dupuy AM, 2001 & France & NR & NR & $22 / 50$ & 44.0 & $4 / 50$ & 8.0 \\
\hline Lambert JC, 1999 & France & NR & 72 & $348 / 600$ & 58.0 & NR & NR \\
\hline Araria-Goumidi L, 2002 & France & probable & 72.3 & $276 / 451$ & 61.2 & $64 / 451$ & 14.2 \\
\hline Bickeboller H, 1996 & France & probable & NR & $233 / 417$ & 55.9 & $54 / 417$ & 12.9 \\
\hline Martinez M, 1998 & France & probable & NR & $162 / 290$ & 55.9 & $46 / 290$ & 15.9 \\
\hline Merched A, 1998 & France & probable & 77.9 & $58 / 118$ & 49.2 & NR & NR \\
\hline Wavrant-DeVrieze F, 1997 & France & probable & 77.3 & $79 / 144$ & 54.9 & $16 / 144$ & 11.1 \\
\hline Drzezga A, 2009 & Germany & NR & NR & $18 / 32$ & 56.3 & NR & NR \\
\hline Hong GS, 2009 & Germany & NR & 72.3 & $210 / 363$ & 57.9 & NR & NR \\
\hline Czech C, 1994 & Germany & possible & NR & $23 / 40$ & 57.5 & $6 / 40$ & 15.0 \\
\hline Riemenschneider M, 2002 & Germany & probable & 69.3 & $91 / 207$ & 44.0 & NR & NR \\
\hline Paragh G, 2002 & Hungary & NR & 64.3 & $19 / 30$ & 63.3 & $6 / 30$ & 20.0 \\
\hline Rakonczay Z, 2005 & Hungary & possible & 73.1 & $28 / 64$ & 43.8 & $5 / 64$ & 7.8 \\
\hline Gacia M, 2008 & Poland & probable & 72.1 & $136 / 217$ & 62.7 & NR & NR \\
\hline Klimkowicz-Mrowiec, 2009 & Poland & probable & 75.8 & $173 / 332$ & 52.1 & $21 / 332$ & 6.3 \\
\hline Trebunova M, 2009 & Slovakia & NR & 73.26 & $32 / 69$ & 46.4 & NR & NR \\
\hline Gold G, 2003 & Switzerland & NR & 79.9 & $33 / 82$ & 40.2 & NR & NR \\
\hline MA estimate (95\% CI) & & & & $19(4,188)$ & $\begin{array}{l}51.74 \\
(47.57-55.92)^{* *}\end{array}$ & $9(1,818)$ & $\begin{array}{l}11.85 \\
(9.10-14.60)^{* *}\end{array}$ \\
\hline \multicolumn{8}{|l|}{ Test for heterogeneity } \\
\hline $\mathrm{I}^{2}$ & & & & $80.00 \%$ & & $61.00 \%$ & \\
\hline Square-root Tau & & & & 8.08 & & 3.09 & \\
\hline Q p value & & & & $<0.001$ & & 0.009 & \\
\hline
\end{tabular}


Table 2 (continued)

\begin{tabular}{|c|c|c|c|c|c|c|c|}
\hline First author, year & Country & $\begin{array}{l}\text { AD case } \\
\text { definition }\end{array}$ & $\begin{array}{l}\text { Mean } \\
\text { age } \\
\text { years }\end{array}$ & $\begin{array}{l}\mathrm{e} 4 /- \\
\mathrm{n} / \mathrm{N}\end{array}$ & $\begin{array}{l}\text { APOE e4/- } \\
\%\end{array}$ & $\begin{array}{l}\mathrm{e} 4 / 4 \\
\mathrm{n} / \mathrm{N}\end{array}$ & $\begin{array}{l}\mathrm{e} 4 / 4 \\
\%\end{array}$ \\
\hline \multicolumn{8}{|l|}{$\begin{array}{l}\text { Southern/Mediterranean } \\
\text { Europe }\end{array}$} \\
\hline Bowirrat A, 2006 & Israel & NR & 82.6 & $20 / 168$ & 11.9 & NR & NR \\
\hline Folin M, 2005 & Italy & NR & 80.3 & $25 / 79$ & 31.6 & $4 / 79$ & 5.1 \\
\hline Ventriglia M, 2005 & Italy & NR & 72.0 & $90 / 227$ & 39.6 & NR & NR \\
\hline Orsitto G, 2007 & Italy & possible & 79.8 & $35 / 82$ & 42.7 & $5 / 82$ & 6.1 \\
\hline Palumbo R, 1997 & Italy & possible & NR & $58 / 140$ & 41.4 & $5 / 140$ & 3.6 \\
\hline Terreni L, 2003 & Italy & possible & 76.4 & $103 / 256$ & 40.2 & NR & NR \\
\hline Altamura C, 2007 & Italy & probable & 74.7 & $24 / 68$ & 35.3 & NR & NR \\
\hline Bosco P, 2007 & Italy & probable & NR & $49 / 109$ & 45.0 & $8 / 109$ & 7.3 \\
\hline Bracco L, 2007 & Italy & probable & 70.9 & $40 / 85$ & 47.1 & $11 / 85$ & 12.9 \\
\hline Flex A, 2006 & Italy & probable & 77.3 & $80 / 183$ & 43.7 & NR & NR \\
\hline Monastero R, 2003 & Italy & probable & 72.0 & $53 / 149$ & 35.6 & NR & NR \\
\hline Orlacchio A, 2002 & Italy & probable & NR & $35 / 135$ & 25.9 & NR & NR \\
\hline Panza F, 2003 & Italy & probable & 71.6 & $15 / 49$ & 30.6 & $2 / 49$ & 4.1 \\
\hline Piccardi M, 2007 & Italy & probable & 76.8 & $56 / 158$ & 35.4 & $4 / 158$ & 2.5 \\
\hline Poli M, 2003 & Italy & probable & 72.0 & $48 / 113$ & 42.5 & NR & NR \\
\hline Scacchi R, 1995 & Italy & probable & 83.5 & $21 / 80$ & 26.3 & $1 / 80$ & 1.3 \\
\hline Spalletta G, 2007 & Italy & probable & NR & $48 / 99$ & 48.5 & $7 / 99$ & 7.1 \\
\hline Venturelli E, 2005 & Italy & probable & NR & $184 / 432$ & 42.6 & NR & NR \\
\hline Zappia M, 2004 & Italy & probable & 71.3 & $64 / 148$ & 43.2 & NR & NR \\
\hline Fernandes M, 1999 & Portugal & probable & 68.2 & $27 / 74$ & 36.5 & $7 / 74$ & 9.5 \\
\hline Blazquez L, 2007 & Spain & NR & 73.5 & $103 / 211$ & 48.8 & NR & NR \\
\hline Clarimon J, 2003 & Spain & possible & 74.8 & $66 / 118$ & 55.9 & $5 / 118$ & 4.2 \\
\hline Deniz-Naranjo MC, 2008 & Spain & probable & NR & $138 / 282$ & 48.9 & NR & NR \\
\hline Ezquerra M, 1997 & Spain & probable & NR & $76 / 174$ & 43.7 & NR & NR \\
\hline Rodriguez-Rodriguez E, 2008 & Spain & probable & 75.7 & $223 / 414$ & 53.9 & NR & NR \\
\hline Vazquez-Higuera JL, 2009 & Spain & probable & 76.8 & $221 / 408$ & 54.2 & NR & NR \\
\hline Smach MA, 2010 & Tunisia & probable & 73.0 & $45 / 93$ & 48.4 & NR & NR \\
\hline Aybeck H, 2007 & Turkey & possible & 73.3 & $24 / 62$ & 38.7 & $2 / 62$ & 3.2 \\
\hline Yokes MB, 2005 & Turkey & possible & 77.5 & $20 / 68$ & 29.4 & $0 / 68$ & 0.0 \\
\hline MA estimate $(95 \% \mathrm{CI})$ & & & & $29(4,664)$ & $\begin{array}{l}40.45 \\
(36.77-44.13)^{* *}\end{array}$ & $13(1,203)$ & $\begin{array}{l}4.56 \\
(2.74-6.38)^{*}\end{array}$ \\
\hline \multicolumn{8}{|l|}{ Test for heterogeneity } \\
\hline $\mathrm{I}^{2}$ & & & & $84.00 \%$ & & $53.00 \%$ & \\
\hline Square-root Tau & & & & 9.11 & & 2.58 & \\
\hline Q p value & & & & $<0.001$ & & 0.012 & \\
\hline
\end{tabular}

* Significant heterogeneity at $\mathrm{p}<0.10 ;{ }^{*}$ significant heterogeneity at $\mathrm{p}<0.01 . \mathrm{NR}=$ Not reported.

\section{APOE e4 and e4/4 Prevalence Estimates}

Summary Estimates

The meta-analyses reported in table 4 are the pooled estimates for APOE e4 carrier prevalence: 48.7\% (95\% confidence interval, CI: 46.5-51.0) and e4/4 prevalence: 9.6\% (95\% CI: 8.4-10.8). Strong evidence of heterogeneity was observed for these overall estimates, and also when stratified by AD case definitions (table 4 ).
Regional Estimates

An overview of the variation across 6 geographic regions is presented graphically in figure 2 . The metaanalysis estimates and tests for heterogeneity are provided in tables 1-3. As has been observed in the general population, the prevalence varied by geographic location, with the lowest regional estimates for the prevalence of e4 carriers in Asia: 41.9\% (95\% CI: 38.5-45.3); e4/4 prevalence: 7.7\% (95\% CI: 5.8-9.6) or Southern Eu- 
Fig. 2. APOE e4 carrier (heterozygotes or homozygotes) and homozygote e4/4 prevalence in the $\mathrm{AD}$ population, stratified by region ( $\mathrm{T}$ bars indicate $95 \% \mathrm{CI}$ ).

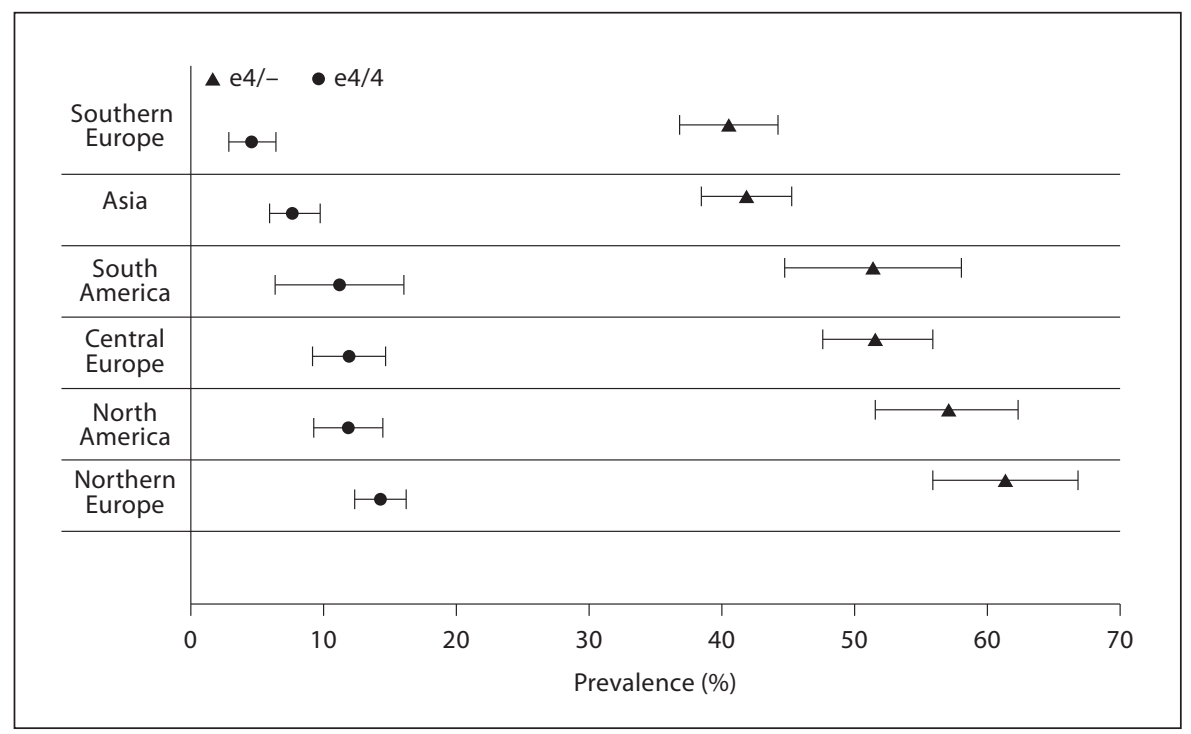

rope/Mediterranean: 40.5\% (95\% CI: 36.8-44.1); e4/4 prevalence: $4.6 \%$ (95\% CI: 2.7-6.4). The highest were in Northern Europe: 61.3\% (95\% CI: 55.9-66.7); e4/4 prevalence: $14.1 \%$ (95\% CI: 12.2-16.0). However, substantial heterogeneity of these prevalence estimates was observed, as indicated by a value of $\mathrm{I}^{2}$ greater than $50 \%$ for each region. The meta-analysis results for each region, stratified by $\mathrm{AD}$ case definition, are provided in table 5 . The probable cases generally had higher estimates within each region, albeit with substantial heterogeneity in the results from each study.

\section{Country-Specific Estimates}

Fifteen countries had more than 3 studies and, for these 15 countries, the data were pooled for each country (table 6). Substantial within-region variation was evident; for example, in Asia the point estimate and 95\% CI for Japan were $48.9 \%$ (95\% CI: 45.9-51.9), and for China they were $32.8 \%$ (95\% CI: 28.3-37.3). Substantial heterogeneity of the majority of these country-specific prevalence estimates was observed, as indicated by a value of $\mathrm{I}^{2}$ greater than $50 \%$ for all the countries except 4 - Canada, France, South Korea, and Spain. Subgroup analyses were conducted to explore sources of heterogeneity by AD case definition (table 7). The probable cases generally had higher estimates within each country, although there was substantial heterogeneity in the study results.

Variation within Countries

Analyses were also conducted to explore sources of heterogeneity within Italy, Japan, and the USA, as ade- quate numbers of studies, $>3$ per area within the country, were available to support exploring estimates obtained from different areas within each of these countries. For example, estimates for Italy from 6 studies (948 patients) conducted in the North (42.51\%; 95\% CI: 39.33-45.68) were similar to 10 studies (1,351 patients) in the South (39.35\%; 95\% CI: 36.42-42.27). For Japan, estimates from 3 studies (349 patients) conducted in the North were higher (55.59\%; 95\% CI: 50.36-60.81) than 13 studies (2,092 patients) in the South $(48.05 \%$; $95 \%$ CI: $44.34-$ 51.76). For the USA, the variation in pooled estimates by location was explored further by assigning studies to 4 groups - the pooled estimates from 5 studies (374 patients) conducted in the West (67.10\%; 95\% CI: 62.2971.91 ) and 3 studies (593 patients) in the South (66.61\%; 95\% CI: 62.81-70.41) were higher than 6 studies $(2,092$ patients) in the Northeast (50.34\%; 95\% CI: 39.63-61.06) and 3 studies (480 patients) in the central states (40.97\%; 95\% CI: 26.48-55.47).

\section{Meta-Regression}

As significant heterogeneity was present, the variation in each geographic region was explored using metaregression. Although meta-regression can adjust for the effect of study level characteristics on the results, the factors should be commonly reported at the study level, and this limits the factors that can be considered in these analyses. In the meta-regression analyses for APOE e4 carrier prevalence in Asia and North America, the AD case definition significantly contributed to the heterogeneity of the regional results, although this was not ob- 
Table 3. Study characteristics and meta-analysis estimates of the prevalence of APOE e 4 carriers (e4/-) and e4/4 in America and Oceania

\begin{tabular}{|c|c|c|c|c|c|c|c|}
\hline First author, year & Country & $\begin{array}{l}\text { AD case } \\
\text { definition }\end{array}$ & $\begin{array}{l}\text { Mean } \\
\text { age } \\
\text { years }\end{array}$ & $\begin{array}{l}\mathrm{e} 4 /- \\
\mathrm{n} / \mathrm{N}\end{array}$ & $\begin{array}{l}\text { APOE e4/- } \\
\%\end{array}$ & $\begin{array}{l}\mathrm{e} 4 / 4 \\
\mathrm{n} / \mathrm{N}\end{array}$ & $\begin{array}{l}\mathrm{e} 4 / 4 \\
\%\end{array}$ \\
\hline \multicolumn{8}{|l|}{ North America } \\
\hline Gauthier E, 2000 & Canada & possible & NR & $41 / 68$ & 60.3 & NR & NR \\
\hline Percy M, 2008 & Canada & probable & 74.1 & $37 / 54$ & 68.5 & $5 / 54$ & 9.3 \\
\hline Wood PL, 2010 & Canada & probable & NR & $26 / 40$ & 65.0 & $9 / 40$ & 22.5 \\
\hline He J, 2009 & USA & $\mathrm{NR}$ & 77.8 & $44 / 68$ & 64.7 & NR & NR \\
\hline Johnston J, 2000 & USA & NR & 82.8 & $29 / 102$ & 28.4 & NR & NR \\
\hline Morris MC, 2000 & USA & NR & NR & $86 / 216$ & 39.8 & NR & NR \\
\hline Sheu KFR, 1999 & USA & NR & 89.0 & $68 / 179$ & 38.0 & NR & NR \\
\hline Zhan J, 2009 & USA & NR & 76.8 & $95 / 181$ & 52.5 & NR & NR \\
\hline Adak S, 2004 & USA & possible & 71.9 & $26 / 39$ & 66.7 & NR & NR \\
\hline Devi G, 1999 & USA & possible & 78.0 & $106 / 312$ & 34.0 & NR & NR \\
\hline Grubber J, 1999 & USA & possible & 72.7 & $164 / 245$ & 66.9 & NR & NR \\
\hline Harwood DB, 2004 & USA & possible & NR & $457 / 960$ & 47.6 & $58 / 960$ & 6.0 \\
\hline Murrell JR, 2006 & USA & possible & 83.8 & $88 / 162$ & 54.3 & $21 / 162$ & 13.0 \\
\hline Nielson K, 1996 & USA & possible & 74.1 & $30 / 42$ & 71.4 & $6 / 42$ & 14.3 \\
\hline Town T, 1998 & USA & possible & NR & $126 / 190$ & 66.3 & $21 / 190$ & 11.1 \\
\hline Weiner MF, 1999 & USA & possible & 72.4 & $105 / 158$ & 66.5 & $20 / 158$ & 12.7 \\
\hline Bretsky PM, 1999 & USA & probable & 80.5 & $57 / 80$ & 71.3 & $6 / 80$ & 7.5 \\
\hline Chuu JYJ, 2006 & USA & probable & NR & $100 / 146$ & 68.5 & NR & NR \\
\hline Dal Forno G, 1996 & USA & probable & NR & 70/101 & 69.3 & $14 / 101$ & 13.9 \\
\hline Gomez-Isla T, 1996 & USA & probable & 77.8 & $222 / 359$ & 61.8 & $59 / 359$ & 16.4 \\
\hline Moceri VM, 2000 & USA & probable & 78.0 & $178 / 393$ & 45.3 & NR & NR \\
\hline Sunderman EE, 2007 & USA & probable & NR & $20 / 38$ & 52.6 & NR & NR \\
\hline Tsai MS, 1994 & USA & probable & 80.5 & $45 / 77$ & 58.4 & $9 / 77$ & 11.7 \\
\hline MA estimate (95\% CI) & & & & $23(4,210)$ & $\begin{array}{l}56.83 \\
(51.45-62.21)^{* *}\end{array}$ & $11(2,223)$ & $\begin{array}{l}11.82 \\
(9.18-14.46)^{* *}\end{array}$ \\
\hline \multicolumn{8}{|l|}{ Test for heterogeneity } \\
\hline & & & & $91.00 \%$ & & $78.00 \%$ & \\
\hline Square-root Tau & & & & 12.26 & & 3.28 & \\
\hline $\mathrm{Q} p$ value & & & & $<0.001$ & & $<0.001$ & \\
\hline \multicolumn{8}{|l|}{ South America } \\
\hline Souza DRS, 2003 & Brazil & NR & 71.5 & $31 / 68$ & 45.6 & $3 / 68$ & 4.4 \\
\hline Bahia VS, 2008 & Brazil & probable & 75.2 & $61 / 120$ & 50.8 & $13 / 120$ & 10.8 \\
\hline Quiroga P, 1999 & Chile & possible & NR & $59 / 95$ & 62.1 & $17 / 95$ & 17.9 \\
\hline Jacquier M, 2001 & Colombia & possible & 73.3 & $32 / 83$ & 38.6 & $7 / 83$ & 8.4 \\
\hline Arboleda GH, 2001 & Colombia & probable & 72.4 & $35 / 61$ & 57.4 & $10 / 61$ & 16.4 \\
\hline Forero DA, 2006 & Colombia & probable & 73.3 & $56 / 106$ & 52.8 & NR & NR \\
\hline Jacquier M, 2001 & Colombia & probable & NR & $19 / 39$ & 48.7 & $5 / 39$ & 12.8 \\
\hline MA estimate (95\% CI) & & & & $6(533)$ & $\begin{array}{l}51.29 \\
(44.66-57.92)^{*}\end{array}$ & $5(427)$ & $\begin{array}{l}11.14 \\
(6.34-15.95)^{*}\end{array}$ \\
\hline \multicolumn{8}{|l|}{ Test for heterogeneity } \\
\hline $\mathrm{I}^{2}$ & & & & $57.00 \%$ & & $57.00 \%$ & \\
\hline Square-root Tau & & & & 6.32 & & 4.29 & \\
\hline $\mathrm{Q} p$ value & & & & 0.039 & & 0.055 & \\
\hline
\end{tabular}


Table 3 (continued)

\begin{tabular}{|c|c|c|c|c|c|c|c|}
\hline First author, year & Country & $\begin{array}{l}\text { AD case } \\
\text { definition }\end{array}$ & $\begin{array}{l}\text { Mean } \\
\text { age } \\
\text { years }\end{array}$ & $\begin{array}{l}\mathrm{e} 4 /- \\
\mathrm{n} / \mathrm{N}\end{array}$ & $\begin{array}{l}\text { APOE e4/- } \\
\%\end{array}$ & $\begin{array}{l}\mathrm{e} 4 / 4 \\
\mathrm{n} / \mathrm{N}\end{array}$ & $\begin{array}{l}\mathrm{e} 4 / 4 \\
\%\end{array}$ \\
\hline \multicolumn{8}{|l|}{ Oceania } \\
\hline Yang JG, 1996 & Australia & NR & NR & $26 / 30$ & 86.7 & $6 / 30$ & 20.0 \\
\hline Ellis KA, 2009 & Australia & possible & 78 & $133 / 211$ & 63.0 & NR & NR \\
\hline Martins RN, 1995 & Australia & probable & NR & $72 / 142$ & 50.7 & $19 / 142$ & 13.4 \\
\hline Taddei K, 2002 & Australia & probable & NR & $147 / 280$ & 52.5 & $19 / 280$ & 6.8 \\
\hline MA estimate (95\% CI) & & & & $4(663)$ & $\begin{array}{l}62.44 \\
(47.23-77.65)^{* *}\end{array}$ & $3(452)$ & $\begin{array}{l}11.61 \\
(4.88-18.34)^{*}\end{array}$ \\
\hline \multicolumn{8}{|l|}{ Test for heterogeneity } \\
\hline $\mathrm{I}^{2}$ & & & & $84.00 \%$ & & $77.00 \%$ & \\
\hline Square-root Tau & & & & 14.84 & & 4.88 & \\
\hline Q p value & & & & $<0.001$ & & 0.014 & \\
\hline
\end{tabular}

* Significant heterogeneity at $\mathrm{p}<0.10$;* significant heterogeneity at $\mathrm{p}<0.01$.

Table 4. APOE e4 carrier and homozygote (e4/4) prevalence, percentage, and $95 \%$ CI in the AD population combined estimate, stratified by $\mathrm{AD}$ case definition

\begin{tabular}{lllllll}
\hline Outcome & AD population & $\begin{array}{l}\text { Studies, } \\
\text { (patients) }\end{array}$ & $\begin{array}{l}\text { Prevalence, } \% \\
(95 \% \mathrm{CI})\end{array}$ & $\mathrm{I}^{2}, \%$ & $\begin{array}{l}\text { Square-root } \\
\text { Tau }\end{array}$ & $\begin{array}{l}\mathrm{Q} \\
\text { p value }\end{array}$ \\
\hline APOE e4/- & All studies & $142(27,109)$ & $48.74(46.50-50.98)^{*}$ & 92.00 & 12.81 & $<0.001$ \\
& Possible & $66(11,375)$ & $46.83(43.25-50.41)^{*}$ & 93.00 & 14.01 & $<0.001$ \\
& Probable & $77(15,773)$ & $50.38(47.64-53.12)^{*}$ & 91.00 & 11.42 & $<0.001$ \\
\hline APOE e4/4 & All studies & $73(13,378)$ & $9.62(8.43-10.81)^{*}$ & 74.00 & 4.4 & $<0.001$ \\
& Possible & $34(5,551)$ & $8.87(7.09-10.65)^{*}$ & 76.00 & 4.52 & $<0.001$ \\
& Probable & $40(7,866)$ & $10.34(8.78-11.90)^{*}$ & 71.00 & 4.15 & $<0.001$ \\
\end{tabular}

* Significant heterogeneity at $\mathrm{p}<0.01$.

served for all regions. In the analyses of North America, AD case definition, community studies, and age $\leq 75$ years contributed to the heterogeneity. None of the variables included in these analyses consistently explained the heterogeneity among all the APOE e4 regional results.

As significant heterogeneity was present, the variation in estimates from Italy, Japan, and the USA was explored using meta-regression and included location within a country (e.g. North vs. South). None of the variables for different areas within a country included in these analyses explained the heterogeneity among the results in these 3 countries.

Prevalence of Apolipoprotein E4
In the meta-regression analyses for APOE e4/4, none of the variables included in these analyses explained the heterogeneity in any of the geographic regions or in Italy, Japan, and the USA.

\section{Discussion}

This study summarizes the APOE e4/4 homozygote and e4/- carrier prevalence data published since 1985, and pools the genetic data collected from 27,109 patients diagnosed with $\mathrm{AD}$ residing in 33 countries. Prevalence estimates are reported for $\mathrm{AD}$ populations worldwide, including populations residing in Europe (North, Central, 
Table 5. APOE e4 carrier and homozygote e $4 / 4$ prevalence and 95\% CI, stratified by region and AD case definition

\begin{tabular}{|c|c|c|c|c|}
\hline \multirow[t]{2}{*}{ Region } & \multicolumn{2}{|l|}{ APOE e4/- } & \multicolumn{2}{|l|}{ APOE e $4 / 4$} \\
\hline & studies, $\mathrm{n}$ (patients) & prevalence, $\%$ (95\% CI) & studies, $\mathrm{n}$ (patients) & prevalence, \% (95\% CI) \\
\hline Asia & $43(8,288)$ & $41.88(38.48-45.27)^{* *}$ & $21(3,817)$ & $7.70(5.84-9.55)^{* *}$ \\
\hline Possible & $17(2,797)$ & $36.17(31.30-41.03)^{* *}$ & $8(1,028)$ & $6.14(3.63-8.64)^{*}$ \\
\hline Probable & $26(5,491)$ & $45.61(41.52-49.69)^{* *}$ & $13(2,789)$ & $8.82(6.26-11.38)^{* *}$ \\
\hline \multicolumn{5}{|l|}{ Europe } \\
\hline Northern & $18(4,563)$ & $61.25(55.85-66.66)^{* *}$ & $11(3,438)$ & $14.10(12.19-16.02)^{*}$ \\
\hline Possible & $10(2,301)$ & $56.60(49.77-63.42)^{* *}$ & $7(2,002)$ & $13.50(10.74-16.25)^{*}$ \\
\hline Probable & $8(2,262)$ & $66.95(59.46-74.44)^{* *}$ & $4(1,436)$ & $14.95(11.69-18.21)^{*}$ \\
\hline Central & $19(4,188)$ & $51.74(47.57-55.92)^{* *}$ & $9(1,818)$ & $11.85(9.10-14.60)^{* *}$ \\
\hline Possible & $10(1,457)$ & $48.82(41.36-56.29)^{* *}$ & $4(184)$ & $11.40(6.29-16.51)$ \\
\hline Probable & $9(2,731)$ & $54.39(50.69-58.09)^{* *}$ & $5(1,634)$ & $11.99(8.58-15.40)^{* *}$ \\
\hline Southern/Mediterranean & $29(4,664)$ & $40.45(36.77-44.13)^{* *}$ & $13(1,203)$ & $4.56(2.74-6.38)^{*}$ \\
\hline Possible & $10(1,411)$ & $37.93(30.21-45.66)^{* *}$ & $6(549)$ & $3.82(2.21-5.43)$ \\
\hline Probable & $19(3,253)$ & $41.96(38.16-45.77)^{* *}$ & $7(654)$ & $5.81(2.80-8.82)^{*}$ \\
\hline \multicolumn{5}{|l|}{ America } \\
\hline North America & $23(4,210)$ & $56.83(51.45-62.21)^{* *}$ & $11(2,223)$ & $11.82(9.18-14.46)^{* *}$ \\
\hline Possible & $14(2,922)$ & $53.60(46.13-61.06)^{* *}$ & $5(1,512)$ & $10.49(7.04-13.95)^{* *}$ \\
\hline Probable & $9(1,288)$ & $62.07(55.75-68.38)^{* *}$ & $6(711)$ & $13.39(9.79-17.00)$ \\
\hline South America & $6(533)$ & $51.29(44.66-57.92)^{*}$ & $5(427)$ & $11.14(6.34-15.95)^{*}$ \\
\hline Possible & $3(246)$ & $48.91(34.94-62.89)^{* *}$ & $3(246)$ & $9.92(2.29-17.56)^{*}$ \\
\hline Probable & $4(326)$ & $52.45(47.02-57.89)$ & $3(220)$ & $12.73(8.30-17.16)$ \\
\hline
\end{tabular}

Regions include studies from the following countries: Asia: China, India, Iran, Japan, Russia, South Korea, Taiwan, Thailand; North America: Canada, USA; South America: Brazil, Chile, Colombia; Central Europe: Austria, Belgium, France, Germany, Hungary, Poland, Slovakia, Switzerland; Northern Europe: Finland, The Netherlands, Norway, Sweden, UK; Southern Europe/Mediterranean: Israel, Italy, Portugal, Spain, Tunisia, Turkey.

* Significant heterogeneity at $\mathrm{p}<0.10$; ${ }^{*}$ significant heterogeneity at $\mathrm{p}<0.01$.

Table 6. APOE e4 carrier and homozygote e4/4 prevalence and 95\% CI, stratified by country

\begin{tabular}{|c|c|c|c|c|}
\hline \multirow[t]{2}{*}{ Country } & \multicolumn{2}{|l|}{ APOE e4/- } & \multicolumn{2}{|l|}{ APOE e4/4 } \\
\hline & studies, $\mathrm{n}$ (patients) & prevalence, $\%$ (95\% CI) & studies, $\mathrm{n}$ (patients) & prevalence, $\%(95 \% \mathrm{CI})$ \\
\hline Australia & $4(663)$ & $62.44(47.23-77.65)^{* *}$ & $3(452)$ & $11.61(4.88-18.34)^{*}$ \\
\hline Canada & $3(162)$ & $64.20(56.79-71.61)$ & - & \\
\hline China & $15(3,125)$ & $32.78(28.26-37.31)^{* *}$ & $4(534)$ & $5.52(1.05-9.99)^{* *}$ \\
\hline Colombia & $3(250)$ & $49.37(38.42-60.32)^{*}$ & - & \\
\hline Finland & $4(689)$ & $64.14(54.87-73.41)^{* *}$ & - & \\
\hline France & $7(2,070)$ & $56.36(53.54-59.18)$ & $5(1,352)$ & $13.61(11.77-15.44)$ \\
\hline Germany & $4(642)$ & $53.04(44.85-61.24)^{*}$ & - & \\
\hline Italy & $18(2,592)$ & $39.08(36.21-41.95)^{* *}$ & $9(881)$ & $5.07(2.99-7.14)^{*}$ \\
\hline Japan & $18(4,147)$ & $48.92(45.91-51.93)^{* *}$ & $9(2,815)$ & $9.69(8.07-11.32)$ \\
\hline The Netherlands & $3(555)$ & $52.04(31.42-72.65)^{* *}$ & $3(555)$ & $12.26(5.33-19.19)^{* *}$ \\
\hline South Korea & $4(450)$ & $46.25(38.95-53.55)$ & $3(134)$ & $2.24(0.00-4.75)$ \\
\hline Spain & $6(1,607)$ & $51.15(47.88-54.41)$ & - & \\
\hline Sweden & $6(1,738)$ & $67.88(58.78-76.99)^{* *}$ & $3(1,417)$ & $15.71(11.36-20.06)^{*}$ \\
\hline UK & $4(1,205)$ & $56.16(50.43-61.90)^{* *}$ & - & \\
\hline USA & $20(4,048)$ & $55.84(49.90-61.78)^{* *}$ & $9(2,129)$ & $11.47(8.74-14.21)^{* *}$ \\
\hline
\end{tabular}

* Significant heterogeneity at $\mathrm{p}<0.10 ;{ }^{* *}$ significant heterogeneity at $\mathrm{p}<0.01$. 
and South/Mediterranean), Asia, North America, and South America. Enough studies have been conducted in 9 countries (Australia, China, France, Germany, Japan, Italy, Spain, the UK, and the USA) to also develop country-specific estimates.

The meta-analysis results suggest that heterogeneity within geographic locations and AD definitions is not fully explained by variation in the source of the patient sample, study design, or mean age of the individual study population. The potential for selection bias has to be considered, as the frequency of the APOE e4 allele has been observed to be lower in community-based AD patient samples than in research clinics, trials, or autopsy studies $[13,14]$. This review excluded trials and autopsy studies, but it should be noted that almost two thirds of the participants included in the analysis were recruited while attending a clinic. This analysis also could not consider the factors influencing why some patients volunteer for this genetic test. There can be genetic heterogeneity within areas of a country, and some studies may select a specific or non-representative population; for example, Bowirrat et al. [15] conducted a door-to-door survey of elderly Arabs residing in a certain area of Israel (Wadi Ara). Although differences in $\mathrm{AD}$ case definitions contribute to the substantial heterogeneity of published prevalence estimates within each region, the variables included in these analyses could not explain the heterogeneity observed in the published estimates. Meta-regression can adjust for the effect of study level characteristics on the results, but relatively few factors were commonly reported at the study level, and this limited the factors able to be considered in this analysis. For example, race was not recorded in many studies, and the ability to fully explore age was limited to the mean age reported in the publications. Although the reasons for the substantial heterogeneity were not possible to elucidate from this review, caution is warranted when attempting to infer regional or country-specific estimates of the ApoE e4 allele frequency. Such differences may arise from small-area variations in allele frequency, environmental risks that vary between regions, methodologic differences, or a combination. The heterogeneity limits the ability to incorporate allele frequencies into models forecasting AD incidence and prevalence estimates and may hinder health policy planning efforts related to allele frequency.

The pooled estimate for APOE e4 carrier prevalence was $48.7 \%$ (95\% CI: 46.5-51.0), and homozygote (e4/4) prevalence was $9.6 \%$ (95\% CI: 8.4-10.8). However, there was substantial heterogeneity in these prevalence estimates. In 1997, Farrer et al. [4] published somewhat high-

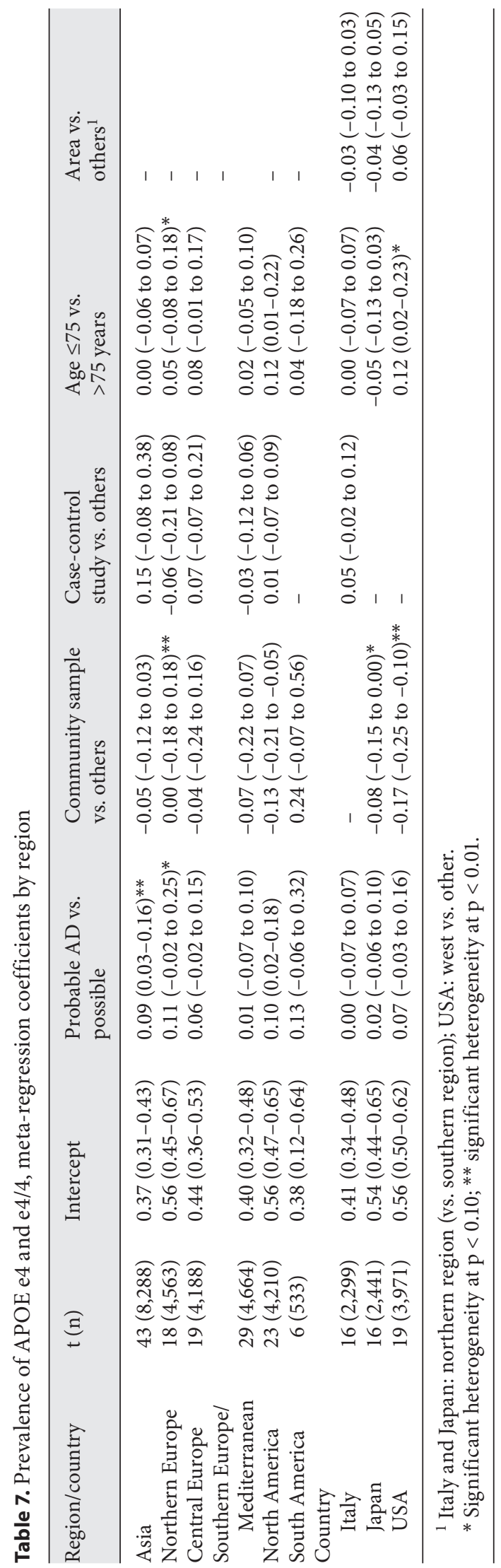


er prevalence estimates (e4/-: $58.5 \%$ and e4/4: $14.8 \%$ ) from a pooled analysis of data, provided by 40 research teams, that had been collected from 5,107 'Caucasian' patients diagnosed with probable or definite AD. The lower estimates in the current study could perhaps be anticipated as approximately one third of the cases are from Asia, where APOE e4 prevalence is lower in the general population, and autopsy studies were excluded.

The lowest regional estimates for e 4 carrier status were observed in Asia (41.9\%; 95\% CI: 38.5-45.3) and Southern Europe (40.5\%; 95\% CI: 36.8-44.1), where the majority of $\mathrm{AD}$ cases were not APOE e4 carriers. In marked contrast, the majority of cases in Northern Europe were carriers (61.3\%; 95\% CI: 55.9-66.7). Similar patterns were observed for APOE e4/4 estimates. These trends are consistent with general population studies in which the APOE e4 frequencies were observed to be higher in Northern than in Southern Europe or Asia, and to be lowest in Italy, Japan and Korea [6-8]. Substantial heterogeneity of the estimates was observed within each region and remained when additional analyses pooled the estimates by country or, in some cases, by areas within each country.

When the analyses were stratified by $\mathrm{AD}$ case definition, graphically there was a general trend within each region for a higher prevalence in the studies selecting 'probable' $\mathrm{AD}$ cases. In the meta-regression analysis for Asia, North America, and Australia, the case definition contributed to the heterogeneity of these regional results, although this was not observed for all regions. For this analysis, the term 'probable' AD case is applied only to the samples specifically reported as meeting the NINCDSADRDA criteria. The differences in AD case definitions are therefore likely to be contributing to the heterogeneity observed in the published prevalence estimates, especially as not all the studies specified the criteria applied. This review identified studies conducted in this field during the last 25 years, and each of the researchers applied the current clinical diagnostic criteria for $\mathrm{AD}$; however, in the future, biomarkers for AD will increasingly be used to support diagnoses in research settings [16], and this has the potential to improve the design of studies in this field.

Genotyping results stratified by familial AD, sporadic $\mathrm{AD}$, early-onset $\mathrm{AD}$, and late-onset $\mathrm{AD}$ were extracted when reported. However, the number of studies reporting these specific categories was insufficient to conduct analyses by region. Although differences in the genetic susceptibility for these different clinical presentations of AD may explain in part the substantial amount of interstudy heterogeneity demonstrated across every analysis, this could not be explored in this project.

Although APOE e4 is a well-studied genetic risk factor for developing $\mathrm{AD}$, in some regions most patients do not carry this genotype, and additional research is needed to be able to understand both other genetic and environmental risk factors. Currently, genetic testing of APOE e4 carrier status is not routinely considered in clinical practice. However, if the mechanism of action of new products for preventing the progression of $\mathrm{AD}$ is contingent on a patient's APOE e4 carrier status, then there may be regions where testing could be essential to consider both during drug development and postmarketing.

\section{Acknowledgment}

This study was sponsored by JANSSEN Alzheimer Immunotherapy and Wyeth (which was acquired by Pfizer in October 2009).

\section{Disclosure Statement}

A. Ward, S. Crean, C.J. Mercaldi, J.M. Collins, and D. Boyd are full-time employees of United BioSource Corporation, which was hired as a vendor by Janssen Alzheimer Immunotherapy. M.N. Cook is a full-time employee of Pfizer Inc. H.M. Arrighi is a fulltime employee of Janssen Alzheimer Immunotherapy.

\section{Appendix}

Included Studies

1 Adak S, Illouz K, Gorman W, Tandon R, Zimmerman EA, Guariglia R, Moore MM, Kaye JA: Predicting the rate of cognitive decline in aging and early Alzheimer disease. Neurology 2004;63:108-114.

2 Ahn Jo S, Ahn K, Kim JH, Kang BH, Kim E, Jo I, Kim DK: APOE-epsilon 4-dependent association of the choline acetyltransferase gene polymorphisms $(2,384 \mathrm{G}>\mathrm{A}$ and $1,882 \mathrm{G}>\mathrm{A})$ with Alzheimer's disease. Clin Chim Acta 2006;368:179-182.

3 Altamura C, Squitti R, Pasqualetti P, Tibuzzi F, Silvestrini M, Ventriglia MC, Cassetta E, Rossini PM, Vernieri F: What is the relationship among atherosclerosis markers, apolipoprotein E polymorphism and dementia? Eur J Neurol 2007; 14:679-682.

4 Andreasen N, Hesse C, Davidsson P, Minthon L, Wallin A, Winblad B, Vanderstichele H, Vanmechelen E, Blennow K: Cerebrospinal fluid beta-amyloid(1-42) in Alzheimer disease: differences between early- and late-onset Alzheimer disease and stability during the course of disease. Arch Neurol 1999;56:673-680.

5 Araria-Goumidi L, Lambert JC, Cottel D, Amouyel P, Chartier-Harlin MC: No association of the HLA-A2 allele with Alzheimer's disease. Neurosci Lett 2002;335:75-78. 
6 Arboleda GH, Yunis JJ, Pardo R, Gomez CM, Hedmont D, Arango G, Arboleda H: Apolipoprotein E genotyping in a sample of Colombian patients with Alzheimer's disease. Neurosci Lett 2001;305:135-138.

7 Asada T, Motonaga T, Kinoshita T: Predictors of severity of behavioral disturbance among community-dwelling elderly individuals with Alzheimer's disease: a 6-year follow-up study. Psychiatry Clin Neurosci 2000;54:673-677.

8 Aybek H, Ercan F, Aslan D, Sahiner T: Determination of malondialdehyde, reduced glutathione levels and APOE4 allele frequency in lateonset Alzheimer's disease in Denizli, Turkey. Clinical Biochemistry 2007;40:172-176.

9 Bahia VS, Kok F, Marie SN, Shinjo SO, Caramelli P, Nitrini R: Polymorphisms of APOE and LRP genes in Brazilian individuals with Alzheimer disease. Alzheimer Dis Assoc Disord 2008;22:61-65.

10 Bettens K, Brouwers N, Engelborghs S, Pooter TD, Deyn PPD, Sleegers $\mathrm{K}$, Broeckhoven CV: DNMBP is genetically associated with Alzheimer dementia in the Belgian population. Neurobiol Aging 2009;30:20002009.

11 Bi XH, Zhao HL, Zhang ZX, Zhang JW: Association of RFC1 A80g and MTHFR C677t polymorphisms with Alzheimer's disease. Neurobiol Aging 2009;30:1601-1607.

12 Bickeboller H, Campion D, Brice A, Amouyel P, Hannequin D, Didierjean O, Penet C, Martin C, Perez-Tur J, Michon A, Dubois B, Ledoze F, Thomas-Anterion C, Pasquier F, Puel M, Demonet JF, Moreaud O, Babron MC, Meulien D, Guez D, Chartier-Harlin MC, Frebourg T, Agid Y, Martinez M, Clerget-Darpoux F: Apolipoprotein E and Alzheimer disease: genotype-specific risks by age and sex. Am J Hum Genet 1997;60:439-446.

13 Blazquez L, Juan DD, Ruiz-Martinez J, Emparanza JI, Saenz A, Otaegui D, Sistiaga A, Martinez-Lage P, Lamet I, Samaranch L, Buiza C, Etxeberria I, Arriola E, Cuadrado E, Urdaneta E, Yanguas J, Munain ALD: Genes related to iron metabolism and susceptibility to Alzheimer's disease in Basque population. Neurobiol Aging 2007;28:1941-1943.

14 Bosco P, Caraci F, Copani A, Spada RS, Sortino MA, Salluzzo R, Salemi M, Nicoletti F, Ferri R: The CDC2 I-G-T haplotype associated with the APOE (epsilon) 4 allele increases the risk of sporadic Alzheimer's disease in Sicily. Neurosci Lett 2007;419:195-198.

15 Bowirrat A, Oscar-Berman M, Logroscino G: Association of depression with Alzheimer's disease and vascular dementia in an elderly Arab population of Wadi-Ara, Israel. Int J Geriatr Psychiatry 2006;21:246-251.

16 Bracco L, Piccini C, Baccini M, Bessi V, Biancucci F, Nacmias B, Bagnoli S, Sorbi S: Pattern and progression of cognitive decline in Alzheimer's disease: role of premorbid intelligence and APOE genotype. Dement Geriatr Cogn Disord 2007;24:483-491.

17 Bretsky PM, Buckwalter JG, Seeman TE, Miller CA, Poirier J, Schellenberg GD, Finch CE, Henderson VW: Evidence for an interaction between apolipoprotein E genotype, gender, and Alzheimer disease. Alzheimer Dis Assoc Disord 1999;13:216-221.

18 Chandak GR, Sridevi MU, Vas CJ, Panikker DM, Singh L: Apolipoprotein $\mathrm{E}$ and presenilin-1 allelic variation and Alzheimer's disease in India. Hum Biol 2002;74:683-693.

19 Chen L, Baum L, Ng HK, Chan LY, Sastre I, Artiga MJ, Valdivieso F, Bullido MJ, Chiu HF, Pang CP: Apolipoprotein E promoter and alpha2macroglobulin polymorphisms are not genetically associated with Chinese late onset Alzheimer's disease. Neurosci Lett 1999;269:173-177.

20 Chuu JY, Taylor JL, Tinklenberg J, Noda A, Yesavage J, Murphy GM Jr: The brain-derived neurotrophic factor VAL66MET polymorphism and rate of decline in Alzheimer's disease. J Alzheimers Dis 2006;9:43-49.

21 Clarimon J, Munoz FJ, Boada M, Tarraga L, Sunyer J, Bertranpetit J, Comas D: Possible increased risk for Alzheimer's disease associated with neprilysin gene. J Neural Transm 2003;110:651-657.

22 Craig D, Hart DJ, McIlroy SP, Passmore AP: Association analysis of apolipoprotein $\mathrm{E}$ genotype and risk of depressive symptoms in $\mathrm{Alz}$ heimer's disease. Dement Geriatr Cogn Disord 2005;19:154-157.

23 Czech C, Forstl H, Hentschel F, Monning U, Besthorn C, GeigerKabisch C, Sattel H, Masters C, Beyreuther K: Apolipoprotein E-4 gene dose in clinically diagnosed Alzheimer's disease: prevalence, plasma cholesterol levels and cerebrovascular change. Eur Arch Psychiatry Clin Neurosci 1994;243:291-292.

24 Davidson Y, Gibbons L, Pritchard A, Hardicre J, Wren J, Stopford C, Julien C, Thompson J, Payton A, Pickering-Brown SM, Pendleton N, Horan MA, Burns A, Purandare N, Lendon CL, Neary D, Snowden JS, Mann DMA: Apolipoprotein E (epsilon) 4 allele frequency and age at onset of Alzheimer's disease. Dement Geriatr Cogn Disord 2007;23:60-66.

25 Deniz-Naranjo MC, Munoz-Fernandez C, Alemany-Rodriguez MJ, Perez-Vieitez MC, Aladro-Benito Y, Irurita-Latasa J, Sanchez-Garcia F: Cytokine IL-1 beta but not IL-1 alpha promoter polymorphism is associated with Alzheimer disease in a population from the Canary Islands, Spain. Eur J Neurol 2008;15:1080-1084.

26 Devi G, Ottman R, Tang M, Marder K, Stern Y, Tycko B, Mayeux R: Influence of APOE genotype on familial aggregation of AD in an urban population. Neurology 1999;53:789-794.

27 Drzezga A, Grimmer T, Henriksen G, Muhlau M, Perneczky R, Miederer I, Praus C, Sorg C, Wohlschlager A, Riemenschneider M, Wester HJ, Foerstl H, Schwaiger M, Kurz A: Effect of APOE genotype on amyloid plaque load and gray matter volume in Alzheimer disease. Neurology 2009;72:1487-1494.

28 Dupuy AM, Badiou S, Ritchie K, Mas E, Descomps B, Cristol JP, Touchon J: Discrepancies between apolipoprotein E phenotyping and genotyping in the elderly. Clin Chem Lab Med 2001;39:405-413.

29 Ellis KA, Bush AI, Darby D, Fazio DD, Foster J, Hudson P, Lautenschlager NT, Lenzo N, Martins RN, Maruff P, Masters C, Milner A, Pike K, Rowe C, Savage G, Szoeke C, Taddei K, Villemagne V, Woodward M, Ames D, Group AR: The Australian imaging, biomarkers and lifestyle (AIBL) study of aging: methodology and baseline characteristics of 1,112 individuals recruited for a longitudinal study of Alzheimer's disease. Int Psychogeriatr 2009;21:672-687.

30 Ezquerra M, Blesa R, Tolosa E, Pousa SL, Aguilar M, Pena J, Broeckhoven CV, Ballesta F, Oliva R: The genotype 2/2 of the presenilin-1 polymorphism is decreased in Spanish early-onset Alzheimer's disease. Neurosci Lett 1997;227:201-204.

31 Fernandes MA, Oliveira CR, Oliveira LM, Nogueira AJ, Santiago B, Santana I: Apolipoprotein E epsilon4 allele is a risk factor for Alzheimer's disease: the central region of Portugal (Coimbra) as a case study. Eur Neurol 1999;42:183-184.

32 Flex A, Gaetani E, Proia AS, Pecorini G, Straface G, Biscetti F, Fioroni G, Sabusco A, Flore R, Tondi P, Pola P, Pola R: Analysis of functional polymorphisms of metalloproteinase genes in persons with vascular dementia and Alzheimer's disease. J Gerontol A Biol Sci Med Sci 2006; 61:1065-1069.

33 Folin M, Baiguera S, Gallucci M, Conconi MT, Liddo RD, Zanardo A, Parnigotto PP: A cross-sectional study of homocysteine-, no-levels, and CT-findings in Alzheimer dementia, vascular dementia and controls. Biogerontology 2005;6:255-260.

34 Forero DA, Arboleda G, Yunis JJ, Pardo R, Arboleda H: Association study of polymorphisms in LRP1, Tau and 5-HTT genes and Alzheimer's disease in a sample of Colombian patients. J Neural Transm 2006; 113:1253-1262.

35 Forno GD, Rasmusson DX, Brandt J, Carson KA, Brookmeyer R, Troncoso J, Kawas CH: Apolipoprotein E genotype and rate of decline in probable Alzheimer's disease. Arch Neurol 1996;53:345-350.

36 Gacia M, Safranow K, Gabryelewicz T, Styczynska M, Peplonska B, Dziedziejko V, Jakubowska K, Chlubek D, Zekanowski C, Barcikowska M: Two polymorphisms of presenilin-2 gene (PSEN2) 5' regulatory region are not associated with Alzheimer's disease (AD) in the Polish population. J Neural Transm 2008;115:85-90.

37 Gauthier E, Fortier I, Courchesne F, Pepin P, Mortimer J, Gauvreau D: Aluminum forms in drinking water and risk of Alzheimer's disease. Environ Res 2000;84:234-246.

38 Gold G, Blouin JL, Herrmann FR, Michon A, Mulligan R, Sail GD, Bouras C, Giannakopoulos P, Antonarakis SE: Specific BACE1 genotypes provide additional risk for late-onset Alzheimer disease in APOE epsilon 4 carriers. Am J Med Genet B Neuropsychiatr Genet 2003; 119B:44-47. 
39 Gomez-Isla T, West HL, Rebeck GW, Harr SD, Growdon JH, Locascio JJ, Perls TT, Lipsitz LA, Hyman BT: Clinical and pathological correlates of apolipoprotein E epsilon 4 in Alzheimer's disease. Ann Neurol 1996; 39:62-70.

40 Grubber JM, Saunders AM, Crane-Gatherum AR, Scott WK, Martin ER, Haynes CS, Conneally PM, Small GW, Roses AD, Haines JL, Pericak-Vance MA: Analysis of association between Alzheimer disease and the K variant of butyrylcholinesterase (BCHE-K). Neurosci Lett 1999; 269:115-119.

41 Grunblatt E, Zehetmayer S, Bartl J, Loffler C, Wichart I, Rainer MK, Jungwirth S, Bauer P, Danielczyk W, Tragl KH, Riederer P, Fischer P: Genetic risk factors and markers for Alzheimer's disease and/or depression in the VITA study. J Psychiatr Res 2009;43:298-308.

42 Harwood DG, Barker WW, Ownby RL, George-Hyslop PS, Mullan M, Duara R: Apolipoprotein E polymorphism and age of onset for Alzheimer's disease in a bi-ethnic sample. Int Psychogeriatr 2004;16:317326.

43 He J, Farias S, Martinez O, Reed B, Mungas D, DeCarli C: Differences in brain volume, hippocampal volume, cerebrovascular risk factors, and apolipoprotein E4 among mild cognitive impairment subtypes. Arch Neurol 2009;66:1393-1399.

$44 \mathrm{He}$ J, Gui JH, Zhang YH, Yu WZ, Chou DH, Xu JT, Song YB: Relationship between apolipoprotein $\mathrm{E}$ gene and the risk for onset of Alzheimer disease in aged adults in Urumqi. Chin J Clin Rehab 2005;9:207-209.

45 Holmes C, Levy R, McLoughlin DM, Powell JF, Lovestone S: Apolipoprotein E: non-cognitive symptoms and cognitive decline in late onset Alzheimer's disease. J Neurol Neurosurg Psychiatry 1996;61:580-583.

46 Hong GS, Heun R, Jessen F, Popp J, Hentschel F, Kelemen P, Schulz A, Maier W, Kolsch H: Gene variations in GSTM3 are a risk factor for Alzheimer's disease. Neurobiol Aging 2009;30:691-696.

$47 \mathrm{Hu}$ J, Miyatake F, Aizu Y, Nakagawa H, Nakamura S, Tamaoka A, Takahashi R, Urakami K, Shoji M: Angiotensin-converting enzyme genotype is associated with Alzheimer disease in the Japanese population. Neurosci Lett 1999;277:65-67.

$48 \mathrm{Hu}$ JL, Li G, Zhou DX, Zou YX, Zhu ZS, Xu RX, Jiang XD, Zeng YJ: Genetic analysis of interleukin-1A C(-889)T polymorphism with Alzheimer disease. Cell Mol Neurobiol 2009;29:81-85.

49 Huang HM, Kuo YM, Ou HC, Lin CC, Chuo LJ: Apolipoprotein E polymorphism in various dementias in Taiwan Chinese population. J Neural Transm 2002;109:1415-1421.

50 Isoe K, Urakami K, Ji Y, Adachi Y, Nakashima K: Presenilin-1 polymorphism in patients with Alzheimer's disease, vascular dementia and alcohol-associated dementia in Japanese population. Acta Neurol Scand 1996;94:326-328.

51 Jacquier M, Arango D, Villareal E, Torres O, Serrano ML, Cruts M, Montanes P, Cano C, Rodriguez MN, Serneels S, Broeckhoven CV: APOE epsilon 4 and Alzheimer's disease: positive association in a Colombian clinical series and review of the Latin-American studies. Arq Neuropsiquiatr 2001;59:11-17.

52 Jiang H, Jia J: Association between NR2B subunit gene (GRIN2B) promoter polymorphisms and sporadic Alzheimer's disease in the North Chinese population. Neurosci Lett 2009;450:356-360.

53 Johnston JM, Nazar-Stewart V, Kelsey SF, Kamboh MI, Ganguli M: Relationships between cerebrovascular events, APOE polymorphism and Alzheimer's disease in a community sample. Neuroepidemiology 2000;19:320-326.

54 Kawamata J, Tanaka S, Shimohama S, Ueda K, Kimura J: Apolipoprotein E polymorphism in Japanese patients with Alzheimer's disease or vascular dementia. J Neurol Neurosurg Psychiatry 1994;57:1414-1416.

55 Kim HC, Kim DK, Choi IJ, Kang KH, Yi SD, Park J, Park YN: Relation of apolipoprotein E polymorphism to clinically diagnosed Alzheimer's disease in the Korean population. Psychiatry Clin Neurosci 2001;55: $115-120$.

56 Kim JM, Shin IS, Yoon JS: Apolipoprotein E among Korean Alzheimer's disease patients in community-dwelling and hospitalized elderly samples. Dement Geriatr Cogn Disord 2002;13:119-124.
57 Kimura M, Matsushita S, Arai H, Matsui T, Yuzuriha T, Higuchi S: No evidence of association between apolipoprotein $\mathrm{E}$ gene regulatory region polymorphism and Alzheimer's disease in Japanese. J Neural Transm 2000;107:1449-1456.

58 Kimura R, Yamamoto M, Morihara T, Akatsu H, Kudo T, Kamino K, Takeda M: SORL1 is genetically associated with Alzheimer disease in a Japanese population. Neurosci Lett 2009;461:177-180.

59 Klimkowicz-Mrowiec A, Marona M, Wolkow P, Maruszak A, Styczynska M, Barcikowska M, Zekanowski C, Szczudlik A, Slowik A: Interleukin-1 gene -511 CT polymorphism and the risk of Alzheimer's disease in a Polish population. Dement Geriatr Cogn Disord 2009;28:461464.

60 Kuusisto J, Koivisto K, Kervinen K, Mykkanen L, Helkala EL, Vanhanen M, Hanninen T, Pyorala K, Kesaniemi YA, Riekkinen P, et al: Association of apolipoprotein E phenotypes with late onset Alzheimer's disease: population based study. BMJ 1994;309:636-638.

61 Kuwano R, Miyashita A, Arai H, Asada T, Imagawa M, Shoji M, Higuchi S, Urakami K, Kakita A, Takahashi H, Tsukie T, Toyabe S, Akazawa $\mathrm{K}$, Kanazawa I, Ihara Y: Dynamin-binding protein gene on chromosome $10 \mathrm{q}$ is associated with late-onset Alzheimer's disease. Hum Mol Genet 2006;15:2170-2182.

62 Lambert JC, Chartier-Harlin MC, Cottel D, Richard F, Neuman E, Guez D, Legrain S, Berr C, Amouyel P, Helbecque N: Is the LDL receptor-related protein involved in Alzheimer's disease? Neurogenetics 1999;2:109-113.

63 Landen M, Hesse C, Fredman P, Regland B, Wallin A, Blennow K: Apolipoprotein E in cerebrospinal fluid from patients with Alzheimer's disease and other forms of dementia is reduced but without any correlation to the APOE4 isoform. Dementia 1996;7:273-278.

64 Lilius L, Fabre SF, Basun H, Forsell C, Axelman K, Mattila K, Andreadis A, Viitanen M, Winblad B, Fratiglioni L, Lannfelt L: Tau gene polymorphisms and apolipoprotein E epsilon4 may interact to increase risk for Alzheimer's disease. Neurosci Lett 1999;277:29-32.

65 Liu HC, Hong CJ, Liu TY, Chi CW, Tsai SJ: Association analysis for the muscarinic M1 receptor genetic polymorphisms and Alzheimer's disease. Dement Geriatr Cogn Disord 2005;19:42-45.

66 Ma SL, Tang NL, Lam LC, Chiu HF: The association between promoter polymorphism of the interleukin-10 gene and Alzheimer's disease. Neurobiol Aging 2005;26:1005-1010.

67 Martinez M, Campion D, Brice A, Hannequin D, Dubois B, Didierjean O, Michon A, Thomas-Anterion C, Puel M, Frebourg T, Agid Y, Clerget-Darpoux F: Apolipoprotein E epsilon4 allele and familial aggregation of Alzheimer disease. Arch Neurol 1998;55:810-816.

68 Martins RN, Clarnette R, Fisher C, Broe GA, Brooks WS, Montgomery P, Gandy SE: APOE genotypes in Australia: roles in early and late onset Alzheimer's disease and Down's syndrome. Neuroreport 1995;6:15131516.

69 Matsubara-Tsutsui M, Yamagata H, Morishima A, Nakura J, Mitsuda N, Kamino K, Kondo I, Miki T: The 4,752 C/T polymorphism in the presenilin 1 gene increases the risk of Alzheimer's disease in apolipoprotein E4 carriers. Intern Med 2002;41:823-828.

70 McIlroy SP, Crawford VL, Dynan KB, McGleenon BM, Vahidassr MD, Lawson JT, Passmore AP: Butyrylcholinesterase $\mathrm{K}$ variant is genetically associated with late onset Alzheimer's disease in Northern Ireland. J Med Genet 2000;37:182-185.

71 Merched A, Xia Y, Papadopoulou A, Siest G, Visvikis S: Apolipoprotein AIV codon 360 mutation increases with human aging and is not associated with Alzheimer's disease. Neurosci Lett 1998;242:117-119.

72 Moceri VM, Kukull WA, Emanuel I, van Belle G, Larson EB: Early-life risk factors and the development of Alzheimer's disease. Neurology 2000;54:415-420.

73 Monastero R, Cefalu AB, Camarda C, Buglino CM, Mannino M, Barbagallo CM, Lopez G, Camarda LK, Travali S, Camarda R, Averna MR: No association between GLU298ASP endothelial nitric oxide synthase polymorphism and Italian sporadic Alzheimer's disease. Neurosci Lett 2003;341:229-232. 
74 Morris MC, Scherr PA, Hebert LE, Bennett DA, Wilson RS, Glynn RJ Evans DA: The cross-sectional association between blood pressure and Alzheimer's disease in a biracial community population of older persons. J Gerontol A Biol Sci Med Sci 2000;55:M130-M136.

75 Muramatsu T, Matsushita S, Arai H, Sasaki H, Higuchi S: Alpha 1-antichymotrypsin gene polymorphism and risk for Alzheimer's disease. J Neural Transm 1996;103:1205-1210.

76 Murrell JR, Price B, Lane KA, Baiyewu O, Gureje O, Ogunniyi A, Unverzagt FW, Smith-Gamble V, Gao S, Hendrie HC, Hall KS: Association of apolipoprotein E genotype and Alzheimer disease in African Americans. Arch Neurol 2006;63:431-434.

77 Namekata K, Imagawa M, Terashi A, Ohta S, Oyama F, Ihara Y: Association of transferrin C2 allele with late-onset Alzheimer's disease. Hum Genet 1997; 101:126-129.

78 Nielson KA, Nolan JH, Berchtold NC, Sandman CA, Mulnard RA, Cotman CW: Apolipoprotein-E genotyping of diabetic dementia patients: is diabetes rare in Alzheimer's disease? J Am Geriatr Soc 1996;44:897904.

79 Orlacchio A, Kawarai T, Polidoro M, Stefani A, George-Hyslop PHS, Bernardi G: Association analysis between Alzheimer's disease and the nicastrin gene polymorphisms. Neurosci Lett 2002;333:115-118.

80 Orsitto G, Seripa D, Panza F, Franceschi M, Cascavilla L, Placentino G, Matera MG, Paris F, Capurso C, Solfrizzi V, Dallapiccola B, Pilotto A: Apolipoprotein $\mathrm{E}$ genotypes in hospitalized elderly patients with vascular dementia. Dement Geriatr Cogn Disord 2007;23:327-333.

81 Palumbo B, Parnetti L, Nocentini G, Cardinali L, Brancorsini S, Ric cardi C, Senin U: Apolipoprotein-E genotype in normal aging, ageassociated memory impairment, Alzheimer's disease and vascular dementia patients. Neurosci Lett 1997;231:59-61.

82 Panza F, Solfrizzi V, Colacicco AM, Basile AM, Introno AD, Capurso C, Sabba M, Capurso S, Capurso A: Apolipoprotein E (APOE) polymorphism influences serum APOE levels in Alzheimer's disease patients and centenarians. Neuroreport 2003;14:605-608.

83 Paragh G, Balla P, Katona E, Seres I, Egerhazi A, Degrell I: Serum paraoxonase activity changes in patients with Alzheimer's disease and vascular dementia. Eur Arch Psychiatry Clin Neurosci 2002;252:63-67.

84 Percy M, Moalem S, Garcia A, Somerville MJ, Hicks M, Andrews D, Azad A, Schwarz P, Zavareh RB, Birkan R, Choo C, Chow V, Dhaliwal S, Duda V, Kupferschmidt AL, Lam K, Lightman D, Machalek K, Mar W, Nguyen F, Rytwinski PJ, Svara E, Tran M, Yeung L, Zanibbi K, Zener R, Ziraldo M, Freedman M: Involvement of APOE E4 and H63D in sporadic Alzheimer's disease in a folate-supplemented Ontario population. J Alzheimers Dis 2008;14:69-84.

85 Piccardi M, Congiu D, Squassina A, Manconi F, Putzu PF, Mereu RM, Chillotti C, Zompo MD: Alzheimer's disease: case-control association study of polymorphisms in ACHE, CHAT, and BCHE genes in a Sardinian sample. Am J Med Genet B Neuropsychiatr Genet 2007; 144B:895-899.

86 Poli M, Gatta LB, Archetti S, Padovani A, Albertini A, Finazzi D: Association analysis between anterior-pharynx defective-1 genes polymorphisms and Alzheimer's disease. Neurosci Lett 2003;350:77-80.

87 Ponomareva NV, Korovaitseva GI, Rogaev EI: EEG alterations in nondemented individuals related to apolipoprotein e genotype and to risk of Alzheimer disease. Neurobiol Aging 2008;29:819-827.

88 Prince JA, Zetterberg H, Andreasen N, Marcusson J, Blennow K: APOE epsilon4 allele is associated with reduced cerebrospinal fluid levels of Abeta42. Neurology 2004;62:2116-2118.

89 Quan W, Yasuda M, Hashimoto M, Yamamoto Y, Ishii K, Kazui H, Mori E, Kakigi T, Maeda K: Polymorphism of the regulatory region of the presenilin-2 gene in sporadic Alzheimer's disease: a case-control study. J Neurol Sci 2006;240:71-75.

90 Quiroga P, Calvo C, Albala C, Urquidi J, Santos JL, Perez H, Klaassen G: Apolipoprotein E polymorphism in elderly Chilean people with Alzheimer's disease. Neuroepidemiology 1999;18:48-52.

91 Rakonczay Z, Horvath Z, Juhasz A, Kalman J: Peripheral cholinergic disturbances in Alzheimer's disease. Chem Biol Interact 2005;157-158: 233-238.
92 Raygani AV, Rahimi Z, Kharazi H, Tavilani H, Pourmotabbed T: Association between apolipoprotein E polymorphism and serum lipid and apolipoprotein levels with Alzheimer's disease. Neurosci Lett 2006;408:68-72.

93 Riemenschneider M, Schwarz S, Wagenpfeil S, Diehl J, Muller U, Forstl H, Kurz A: A polymorphism of the brain-derived neurotrophic factor (BDNF) is associated with Alzheimer's disease in patients lacking the apolipoprotein E epsilon4 allele. Mol Psychiatry 2002;7:782785 .

94 Rodriguez-Rodriguez E, Llorca J, Mateo I, Infante J, Sanchez-Quintana C, Garcia-Gorostiaga I, Fernandez-Viadero C, Pena N, Berciano $\mathrm{J}$, Combarros O: No association of genetic variants of liver $\mathrm{X}$ receptorbeta with Alzheimer's disease risk. Am J Med Genet B Neuropsychiatr Genet 2008;147B:650-653.

95 Sando SB, Melquist S, Cannon A, Hutton ML, Sletvold O, Saltvedt I, White LR, Lydersen S, Aasly JO: APOE (epsilon)4 lowers age at onset and is a high risk factor for Alzheimer's disease; a case control study from central Norway. BMC Neurol 2008;8:9.

96 Scacchi R, Bernardini LD, Mantuano E, Donini LM, Vilardo T, Corbo RM: Apolipoprotein E (APOE) allele frequencies in late-onset sporadic Alzheimer's disease (AD), mixed dementia and vascular dementia: lack of association of epsilon 4 allele with AD in Italian octogenarian patients. Neurosci Lett 1995;201:231-234.

97 Senanarong V, Harnphadungkit K, Lertrit P, Mitrpant C, Udompunthurak S, Limwong C, Prayoonwiwat N, Poungvarin N: Experience of APOE study in Thai elderly. J Med Assoc Thai 2001;84:182-187.

98 Sheu KF, Brown AM, Haroutunian V, Kristal BS, Thaler H, Lesser M, Kalaria RN, Relkin NR, Mohs RC, Lilius L, Lannfelt L, Blass JP: Modulation by DLST of the genetic risk of Alzheimer's disease in a very elderly population. Ann Neurol 1999;45:48-53.

99 Shi J, Zhang S, Tang M, Liu X, Li T, Wang Y, Han H, Guo Y, Hao Y, Zheng K, Kong X, Su Z, Tong Y, Ma C: The 1239G/C polymorphism in exon 5 of BACE1 gene may be associated with sporadic Alzheimer's disease in Chinese Hans. Am J Med Genet B Neuropsychiatr Genet 2004;124B:54-57.

100 Shibata N, Ohnuma T, Baba H, Higashi S, Nishioka K, Arai H: Genetic association between sorll polymorphisms and Alzheimer's disease in a Japanese population. Dement Geriatr Cogn Disord 2008;26: 161-164.

101 Slooter AJ, de Knijff P, Hofman A, Cruts M, Breteler MM, Van Broeckhoven C, Havekes LM, Duijn CMv: Serum apolipoprotein E level is not increased in Alzheimer's disease: the Rotterdam Study. Neurosci Lett 1998;248:21-24.

102 Smach MA, Charfeddine B, Othman LB, Lammouchi T, Ltaief A, Nafati S, Dridi H, Bennamou S, Limem K: -1154G/A and -2578C/A polymorphisms of the vascular endothelial growth factor gene in Tunisian Alzheimer patients in relation to beta-amyloid (1-42) and total tau protein. Neurosci Lett 2010;472:139-142.

103 Souza DR, de Godoy MR, Hotta J, Tajara EH, Brandao AC, Pinheiro Junior S, Tognola WA, dos Santos JE: Association of apolipoprotein E polymorphism in late-onset Alzheimer's disease and vascular dementia in Brazilians. Braz J Med Biol Res 2003;36:919-923.

104 Spalletta G, Bernardini S, Bellincampi L, Federici G, Trequattrini A, Ciappi F, Bria P, Caltagirone C, Bossu P: Glutathione S-transferase P1 and T1 gene polymorphisms predict longitudinal course and age at onset of Alzheimer disease. Am J Geriatr Psychiatry 2007;15:879-887.

105 Sundermann EE, Gilbert PE, Murphy C: Apolipoprotein E epsilon4 genotype and gender: effects on memory. Am J Geriatr Psychiatry 2007; 15:869-878

106 Suzuki Y, Yamamoto S, Umegaki H, Onishi J, Mogi N, Fujishiro H, Iguchi A: Smell identification test as an indicator for cognitive impairment in Alzheimer's disease. Int J Geriatr Psychiatry 2004;19:727-733.

107 Taddei K, Fisher C, Laws SM, Martins G, Paton A, Clarnette RM, Chung C, Brooks WS, Hallmayer J, Miklossy J, Relkin N, GeorgeHyslop PHS, Gandy SE, Martins RN: Association between presenilin-1 GLU318GLY mutation and familial Alzheimer's disease in the Australian population. Mol Psychiatry 2002;7:776-781. 
108 Tanaka N, Goto Y, Akanuma J, Kato M, Kinoshita T, Yamashita F, Tanaka M, Asada T: Mitochondrial DNA variants in a Japanese population of patients with Alzheimer's disease. Mitochondrion 2010;10: 32-37.

109 Terreni L, Fogliarino S, Quadri P, Ruggieri RM, Piccoli F, Tettamanti M, Lucca U, Forloni G: Tumor necrosis factor alpha polymorphism C-850T is not associated with Alzheimer's disease and vascular dementia in an Italian population. Neurosci Lett 2003;344:135-137.

110 Tilvis RS, Strandberg TE, Juva K: Apolipoprotein E phenotypes, dementia and mortality in a prospective population sample. J Am Geriatr Soc 1998;46:712-715.

111 Toji H, Maruyama H, Sasaki K, Nakamura S, Kawakami H: Apolipoprotein E promoter polymorphism and sporadic Alzheimer's disease in a Japanese population. Neurosci Lett 1999;259:56-58.

112 Town T, Paris D, Fallin D, Duara R, Barker W, Gold M, Crawford F, Mullan M: The -491a/t apolipoprotein E promoter polymorphism association with Alzheimer's disease: independent risk and linkage disequilibrium with the known APOE polymorphism. Neurosci Lett 1998;252:95-98.

113 Trebunova M, Slaba E, Habalova V, Gdovinova Z: The role of the $-427 \mathrm{~T} / \mathrm{C}$ apolipoprotein $\mathrm{E}$ promoter polymorphism in the pathogenesis of Alzheimer's disease, vascular dementia and mixed dementia. J Neural Transm 2009;116:339-344.

114 Tsai MS, Tangalos EG, Petersen RC, Smith GE, Schaid DJ, Kokmen E, Ivnik RJ, Thibodeau SN: Apolipoprotein E: risk factor for Alzheimer disease. Am J Hum Genet 1994;54:643-649.

115 Tsai SJ, Hong CJ, Liu HC, Liu TY, Liou YJ: The brain-derived neurotrophic factor gene as a possible susceptibility candidate for Alzheimer's disease in a Chinese population. Dement Geriatr Cogn Disord 2006;21:139-143.

116 Van der Flier WM, Pijnenburg YAL, Schoonenboom NM, Dik MG, Blankenstein MA, Scheltens P: Distribution of APOE genotypes in a memory clinic cohort. Dement Geriatr Cogn Disord 2008;25:433438.

117 van Duijn CM, de Knijff P, Cruts M, Wehnert A, Havekes LM, Hofman A, Broeckhoven CV: Apolipoprotein E4 allele in a populationbased study of early-onset Alzheimer's disease. Nat Genet 1994;7:7478 .

118 Vazquez-Higuera JL, Sanchez-Juan P, Rodriguez-Rodriguez E, Mateo I, Pozueta A, Frank A, Sastre I, Valdivieso F, Berciano J, Bullido MJ, Combarros O: Dyrkla genetic variants are not linked to Alzheimer's disease in a Spanish case-control cohort. BMC Med Genet 2009;10: 129.

119 Ventriglia M, Chiavetto LB, Scassellati C, Squitti R, Binetti G, Ghidoni R, Rossini PM, Gennarelli M: Lack of association between MnSOD gene polymorphism and sporadic Alzheimer's disease. Aging Clin Exp Res 2005;17:445-448.

120 Venturelli E, Galimberti D, Lovati C, Fenoglio C, Scalabrini D, Mari ani C, Forloni G, Bresolin N, Scarpini E: The T-786C NOS3 polymorphism in Alzheimer's disease: association and influence on gene expression. Neurosci Lett 2005;382:300-303.

121 Vepsalainen S, Helisalmi S, Koivisto AM, Tapaninen T, Hiltunen M, Soininen H: Somatostatin genetic variants modify the risk for Alzheimer's disease among Finnish patients. J Neurol 2007;254:15041508.

122 Wang B, Zhou S, Yang Z, Xie YC, Wang J, Zhang P, Lv Z, Zheng C, Ma X: Genetic analysis of tumor necrosis factor-alpha (TNF-alpha) G-308A and saitohin Q7R polymorphisms with Alzheimer's disease. J Neurol Sci 2008;270:148-151.

123 Wavrant-DeVrieze F, Perez-Tur J, Lambert JC, Frigard B, Pasquier F, Delacourte A, Amouyel P, Hardy J, Chartier-Harlin MC: Association between the low density lipoprotein receptor-related protein (LRP) and Alzheimer's disease. Neurosci Lett 1997;227:68-70.
124 Weiner MF, Vega G, Risser RC, Honig LS, Cullum CM, Crumpacker D, Rosenberg RN: Apolipoprotein E epsilon 4, other risk factors, and course of Alzheimer's disease. Biol Psychiatry 1999;45:633-638.

125 Wood PL, Mankidy R, Ritchie S, Heath D, Wood JA, Flax J, Goodenowe DB: Circulating plasmalogen levels and Alzheimer disease assessment scale-cognitive scores in Alzheimer patients. J Psychiatry Neurosci 2010;35:59-62.

126 Yamagata Z, Asada T, Kinoshita A, Zhang Y, Asaka A: Distribution of apolipoprotein E gene polymorphisms in Japanese patients with Alzheimer's disease and in Japanese centenarians. Hum Hered 1997;47: 22-26.

127 Yang JD, Feng GY, Zhang J, Cheung J, Clair DS, He L, Ichimura K: Apolipoprotein E -491 promoter polymorphism is an independent risk factor for Alzheimer's disease in the Chinese population. Neurosci Lett 2003;350:25-28.

128 Yang JG, Poropat RA, Brooks WS, Broe GA, Nicholson GA: Apolipoprotein E genotyping in Alzheimer's disease in an Australian sample. Aust N Z J Med 1996;26:658-661.

129 Yasuda M, Mori E, Kitagaki H, Yamashita H, Hirono N, Shimada K, Maeda K, Tanaka C: Apolipoprotein E epsilon 4 allele and whole brain atrophy in late-onset Alzheimer's disease. Am J Psychiatry 1998;155: 779-784.

130 Yokes MB, Emre M, Harmanci H, Gurvit H, Hanagasi H, Sahin H, Bilgic B, Basak AN: The apolipoprotein E (APOE) genotype in a Turkish population with Alzheimer's disease. Balkan J Med Genet 2005;8: 57-63.

131 Yoshida S, Iwabuchi Y, Numachi Y, Saito H, Yamazaki H, Sakai H, Kimura M, Matsuoka H, Sato M: Clinical features and alterations in the inferior horn sizes in lateral ventricle in Alzheimer's patients with different APOE genotype in Japanese population. Prog Neuropsychopharmacol Biol Psychiatry 2001;25:1377-1384.

132 Yoshizawa T, Yamakawa-Kobayashi K, Hamaguchi H, Shoji S: Alpha1-antichymotrypsin polymorphism in Japanese cases of Alzheimer's disease. J Neurol Sci 1997;152:136-139.

133 Yu JT, Tan L, Ou JR, Zhu JX, Liu K, Song JH, Sun YP: Polymorphisms at the beta2-adrenergic receptor gene influence Alzheimer's disease susceptibility. Brain Res 2008;1210:216-222.

134 Zappia M, Manna I, Serra P, Cittadella R, Andreoli V, Russa AL, Annesi F, Spadafora P, Romeo N, Nicoletti G, Messina D, Gambardella A, Quattrone A: Increased risk for Alzheimer disease with the interaction of MPO and A2M polymorphisms. Arch Neurol 2004;61:341344.

135 Zetterberg M, Landgren S, Andersson ME, Palmer MS, Gustafson DR, Skoog I, Minthon L, Thelle DS, Wallin A, Bogdanovic N, Andreasen N, Blennow K, Zetterberg H: Association of complement factor $\mathrm{H}$ Y402H gene polymorphism with Alzheimer's disease. Am J Med Genet B Neuropsychiatr Genet 2008;147:720-726.

136 Zhan J, Brys M, Glodzik L, Tsui W, Javier E, Wegiel J, Kuchna I, Pirraglia E, Li Y, Mosconi L, Louis LAS, Switalski R, Santi SD, Kim BC, Wisniewski T, Reisberg B, Bobinski M, Leon MJD: An entorhinal cortex sulcal pattern is associated with Alzheimer's disease. Hum Brain Mapp 2009;30:874-882.

137 Zhang JW, Li XQ, Zhang ZX, Chen D, Zhao HL, Wu YN, Qu QM: Association between angiotensin-converting enzyme gene polymorphism and Alzheimer's disease in a Chinese population. Dement Geriatr Cogn Disord 2005;20:52-56.

138 Zhao FG, Wang YH, Yang JF, Ma QL, Tang Z, Dong XM, Chan P: Association between acyl-coenzyme A: cholesterol acyltransferase gene and risk for Alzheimer's disease in Chinese. Neurosci Lett 2005;388: $17-20$.

139 Zhou S, Huriletemuer, Wang J, Zhang C, Zhao S, de SW, Wang B, Ma $\mathrm{X}$ : Absence of association on aldehyde dehydrogenase 2 (ALDH2) polymorphism with Mongolian Alzheimer patients. Neurosci Lett 2010;468:312-315. 


\section{References}

$>1$ Lobo A, Launer LJ, Fratiglioni L, Andersen K, Di Carlo A, Breteler MM, Copeland JR, Dartigues JF, Jagger C, Martinez-Lage J, Soininen H, Hofman A: Prevalence of dementia and major subtypes in Europe: a collaborative study of population-based cohorts. Neurologic Diseases in the Elderly Research Group. Neurology 2000;54:S4-S9.

$\checkmark 2$ Davidson Y, Gibbons L, Pritchard A, Hardicre J, Wren J, Stopford C, Julien C, Thompson J, Payton A, Pickering-Brown SM, Pendleton N, Horan MA, Burns A, Purandare N, Lendon CL, Neary D, Snowden JS, Mann DM: Apolipoprotein E epsilon4 allele frequency and age at onset of Alzheimer's disease. Dement Geriatr Cogn Disord 2007;23: 60-66.

$\$ 3$ Roses AD: On the discovery of the genetic association of apolipoprotein E genotypes and common late-onset Alzheimer disease. J Alzheimers Dis 2006;9:361-366.

-4 Farrer LA, Cupples LA, Haines JL, Hyman B, Kukull WA, Mayeux R, Myers RH, PericakVance MA, Risch N, van Duijn CM: Effects of age, sex, and ethnicity on the association between apolipoprotein $\mathrm{E}$ genotype and Alzheimer disease. A meta-analysis. APOE and Alzheimer disease meta analysis consortium. JAMA 1997;278:1349-1356.
Bettens K, Sleegers K, Van Broeckhoven C: Current status on Alzheimer disease molecular genetics: from past, to present, to future. Hum Mol Genet 2010;19:R4-R11.

6 Corbo RM, Scacchi R: Apolipoprotein E (APOE) allele distribution in the world. Is $\mathrm{APOE}^{*} 4$ a 'thrifty' allele? Ann Hum Genet 1999;63:301-310.

7 Eichner JE, Dunn ST, Perveen G, Thompson DM, Stewart KE, Stroehla BC: Apolipoprotein E polymorphism and cardiovascular disease: a huge review. Am J Epidemiol 2002. 155:487-495.

-8 Singh PP, Singh M, Mastana SS: APOE distribution in world populations with new data from India and the UK. Ann Hum Biol 2006; 33:279-308.

$\checkmark 9$ DerSimonian R, Laird N: Meta-analysis in clinical trials. Control Clin Trials 1986; 177-188.

10 Hedges L, Olkin I: Statistical Methods for Meta-Analysis. Orlando, Academic Press, 1985.

11 Higgins JP, Thompson SG, Deeks JJ, Altman DG: Measuring inconsistency in meta-analyses. BMJ 2003;327:557-560.

12 Berkey CS, Hoaglin DC, Mosteller F, Colditz GA: A random-effects regression model for meta-analysis. Stat Med 1995;14:395-411.
13 Tsuang D, Kukull W, Sheppard L, Barnhart RL, Peskind E, Edland SD, Schellenberg G, Raskind M, Larson EB: Impact of sample selection on APOE epsilon 4 allele frequency: a comparison of two Alzheimer's disease samples. J Am Geriatr Soc 1996;44:704-707.

14 Crean S, Ward A, Mercaldi CJ, Collins JM, Cook MN, Baker NL, Arrighi HM: Apolipoprotein E e4 prevalence in Alzheimer's disease patients varies across global populations: a systematic literature review and meta-analysis. Dement Geriatr Cogn Disord, 2011;31:20-30.

15 Bowirrat A, Oscar-Berman M, Logroscino G: Association of depression with Alzheimer's disease and vascular dementia in an elderly Arab population of Wadi-Ara, Israel. Int J Geriatr Psychiatry 2006;21:246-251.

16 Dubois B, Feldman HH, Jacova C, Dekosky ST, Barberger-Gateau P, Cummings J, Delacourte A, Galasko D, Gauthier S, Jicha G, Meguro K, O’Brien J, Pasquier F, Robert P, Rossor M, Salloway S, Stern Y, Visser PJ, Scheltens P: Research criteria for the diagnosis of Alzheimer's disease: revising the NINCDS-ADRDA criteria. Lancet Neurol 2007;6:734-746. 